Alloys and Compounds

Elsevier Editorial System(tm) for Journal of

Manuscript Draft

Manuscript Number: JALCOM-D-16-04221R1

Title: Hot deformation characterization of lamellar Ti-43Al-2Si alloy

fabricated by cold crucible continuous casting

Article Type: Full Length Article

Keywords: Titanium aluminum; Hot deformation; 3D processing map; Dynamic recrystallization.

Corresponding Author: Dr. Rui Zhang, Ph.D.

Corresponding Author's Institution: School of Material Science and Engineering

First Author: Rui Zhang, Ph.D.

Order of Authors: Rui Zhang, Ph.D.; Dongjun Wang, Ph.D.; Shiqiu Liu; Hongsheng Ding; Shijian Yuan

Abstract: In order to investigate the hot deformation behavior of lamellar Ti-43Al-2Si (ato, similarly hereinafter) alloy with equiaxed colonies prepared by cold crucible continuous casting, the isothermal compression tests were carried out at different temperatures in the range of 1100-1250oC using the strain rates between $0.001 \mathrm{~s}-1$ and $1 \mathrm{~s}-1$ with true strain of 0.8. A constitutive equation at elevated temperature and threedimensional processing map of this alloy were established based on flow stress data at different temperatures and strain rates. The microstructures depended on deformation condition were observed by optical microscope (OM), backscattered electron (BSE) and electron backscattered diffraction (EBSD). It was found that the deformation

activation energy (Q) varied with the deformation temperature and strain rate, and average value was $451.15 \mathrm{~kJ} / \mathrm{mol}$ which was much larger than that of the powder metallurgy TiAl-based alloy but smaller than that of the well directionally solidified (DS) microstructure TiAl-based alloy. Based on the processing map, the appropriate hot working domain of this alloy without deformed cracks was determined to be within the temperature window from $11500 \mathrm{C}$ to $12500 \mathrm{C}$ and in the strain rate range of $0.001-0.01 \mathrm{~s}-$ 1. The flow softening feature of the present alloy during deformation was noted and caused by synergetic effect of dynamic recrystallization (DRX), globularization and reorientation of lamellae. In addition, the ZenerHollomom parameter played a key role on the DRX process. 
Dear Editor:

On behalf of my co-authors, I am submitting the enclosed material "Hot deformation characterization of lamellar Ti-43Al-2Si alloy fabricated by cold crucible cold crucible continuous casting" for possible publication to your famous journal "Journal of Alloys and Compounds". We certify that the work described has not been published previously, that it is not under consideration for publication elsewhere and that, if accepted, it will not be published elsewhere in the same form, in English or in any other language, without the written consent of the Publisher.

In this paper, the isothermal compression tests of Ti-43AI-2Si alloy prepared by cold crucible continuous casting were carried out at different temperatures in the range of $1100-1250^{\circ} \mathrm{C}$ using the strain rates between $0.001 \mathrm{~s}^{-1}$ and $1 \mathrm{~s}^{-1}$ with true strain of 0.8 . A constitutive equation at elevated temperature and three-dimensional processing map of this alloy were established based on flow stress data at different temperatures and strain rates. The microstructures depended on deformation condition were observed, which can help to understand the underlying relationships between hot deformation ability and microstructural orientation better, and further accelerate the application of this kind of advanced alloy.

Looking forward to hearing from you soon.

With kind regards,

Yours sincerely,

Rui Zhang

Room 606

National Key Laboratory for Precision Hot Processing of Metals

Harbin Institute of Technology, Harbin 150001, P.R.China

Tel/fax: +86-451-86414761

Email:zhangrui8014@163.com 


\section{Prime Novelty Statement}

This work fabricated the TiAl-based alloy by cold crucible continuous casting, and the billet have excellent hot forming property and is without contamination and defects. It further investigates the hot deformation characterization by building 3D hot processing map and constitutive equations. The best hot forming range of this alloy is ensured. It also explains the variation of flow stress-strain curves at different deformation conditions by microstructure observation. Based on backscattered electron (BSE) and electron back-scattered diffraction (EBSD) profile analyses of the deformation sample, dynamic recrystallization (DRX), globularization and reorientation of lamellae were proposed to illustrate the flow softening at hot deformation processing. The lamellar structure of this alloy could be transferred to near gamma fine microstructure by thermo-mechanical treatment (TMT). Thus, the TiAl-based alloy with excellent properties can be prepared by d crucible continuous casting combined with TMT technique in industrial application. 
Dear Editor Battezzati,

RE: JALCOM-D-16-04221

We would like to thank Journal of Alloys and Compounds for giving us an opportunity to revise our manuscript.

We thank the reviewers for their careful reading and thoughtful comments on previous draft. We have carefully taken their comments into consideration in preparing our revision, which has resulted in a paper that is clearer and more compelling. The following summarizes how we responded to reviewer comments.

Thanks for considering our manuscript.

Best wishes,

Yours sincerely,

Rui Zhang

Revision - authors' response

Reviewer \#1:

1) A few English expressions should be improved, e.g. please change "polar figure" to "pole figure".

Response: As suggested by the reviewer we have corrected the English expressions. For example, "polar figure" has been revised as "pole figure", 
"deforming" has been revised as "deformation", and added "higher than" in the "Material and experimental" section.

2) The authors need to describe the preparation of BSE-SEM specimen in the "Material and experimental" section, e.g. what polishing solution was used in this study?

Response: As requested by the reviewer we have added the preparation of BSE-SEM specimen and what the etched reagent was in the "Material and experimental" section. For example, "The specimens were sectioned parallel to the compression axis and mechanically ground, then polished by electrolytic method in a solution composed of $60 \mathrm{vol} \%$ methanol, $34 \mathrm{vol} \%$ butyl alcohol and 6 vol\% perchloric acid at $30 \mathrm{~V}$ and $-25^{\circ} \mathrm{C}$. The etched reagent was the Kroll's reagent, which was 50vol\% nitric acid, 3vol\% hydrofluoric acid and 92vol\% purified water."

3) In "Material and experimental" section, the parameters of cold crucible continuous casting should be given.

Response: As requested by the reviewer we have introduced the parameters of cold crucible continuous casting in the "Material and experimental" section. For example, "The alloy used in this work was received as a vacuum induction melted Ti-43Al-2Si billet and then was remelted by cold crucible continuous casting under the pulling velocity of $10 \mu \mathrm{m} / \mathrm{s}$ and input power of 55kW."

4) For conclusion (1), the relationship of flow stress and Zener-Hollomom 
parameter should be given in detail.

Response: As suggested by the reviewer we have change the conclusion (1) from "The flow stress is sensitive to the deformation temperature and strain rate, namely the Zener-Hollomom ( $Z$ ) parameter. The peak stress decreases with the decreasing of $Z$ parameter, which indicates that the stress in dominated by the combined effect of the working hardening and softening mechanisms. Moreover, the peak stress appears at a critical strain in the initial deforming state, and the flow softening occurs with further increasing of strain." to "The flow stress is sensitive to the deformation condition, namely deformation temperature and strain rate which is related to the Zener-Hollomom (Z) parameter. The flow stress decreases with the decreasing of $Z$ parameter corresponding to increase of deformation temperature and decrease of strain rate. Moreover, the flow stress appears a peak value in the initial deformation state, and the flow softening occurs with further increasing of strain."

Other revision:

We change the corresponding author from "Rui Zhang" to "Dongjun Wang”, Tel./ fax fron "+86-451-86414751" to "+86-451-86402590", and E-mail address from “zhangrui8014@163.com (Rui Zhang);dongjunwang@hit.edu.cn (Dongjun Wang)" to "dongjunwang@hit.edu.cn". 


\title{
Hot deformation characterization of lamellar Ti-43Al-2Si alloy fabricated by cold crucible
}

\section{continuous casting}

\author{
Rui Zhang ${ }^{a}$, Dongjun Wang ${ }^{\mathrm{b}, c *}$, Shiqiu Liu ${ }^{\mathrm{a}}$, Hongsheng Ding ${ }^{\mathrm{a}, \mathrm{b}}$, Shijian Yuan ${ }^{\mathrm{a}, \mathrm{b}}$ \\ ${ }^{a}$ School of Material Science and Engineering, Harbin Institute of Technology, Harbin 150001, P.R. China \\ ${ }^{\mathrm{b}}$ National Key Laboratory of Precision Hot Processing of Metals, Harbin Institute of Technology, Harbin 150001, P.R. China \\ ${ }^{\mathrm{C}}$ Key Laboratory of Micro-Systems and Micro-Structures Manufacturing, Ministry of Education, Harbin 150001, P.R. China
}

\begin{abstract}
In order to investigate the hot deformation behavior of lamellar Ti-43Al-2Si (at\%, similarly hereinafter) alloy with equiaxed colonies prepared by cold crucible continuous casting, the isothermal compression tests were carried out at different temperatures in the range of $1100-1250^{\circ} \mathrm{C}$ using the strain rates between $0.001 \mathrm{~s}^{-1}$ and $1 \mathrm{~s}^{-1}$ with true strain of 0.8 . A constitutive equation at elevated temperature and threedimensional processing map of this alloy were established based on flow stress data at different temperatures and strain rates. The microstructures depended on deformation condition were observed by optical microscope (OM), backscattered electron (BSE) and electron back-scattered diffraction (EBSD). It was found that the deformation activation energy $(Q)$ varied with the deformation temperature and strain rate, and average value was $451.15 \mathrm{~kJ} / \mathrm{mol}$ which was much larger than that of the powder metallurgy TiAl-based alloy but smaller than that of the well directionally solidified (DS) microstructure TiAl-based alloy. Based on the processing map, the appropriate hot working domain of this alloy without deformed cracks was determined to be within the temperature window from $1150^{\circ} \mathrm{C}$ to $1250^{\circ} \mathrm{C}$ and in the strain rate range of $0.001-0.01 \mathrm{~s}^{-1}$. The flow softening feature of the present alloy during deformation was noted and caused by synergetic effect of dynamic recrystallization (DRX), globularization and reorientation of lamellae. In addition, the Zener-Hollomom parameter played a key role on the DRX process.
\end{abstract}

Keywords: Titanium aluminum; Hot deformation; 3D processing map; Dynamic recrystallization.

\section{Introduction}

Titanium aluminide alloys have been drawn much attention for their potential applications in the automotive, aero-engine and power-plant turbine industries owing to the excellent performances, such as low density, high melting point and good corrosion resistance [1-3]. However, the inherent room temperature brittleness of TiAl-based alloys seriously hinders their extensive applications. To overcome this bottleneck, thermo-mechanical treatment (TMT) [4] is an optional method to improve the ductility of these alloys through

\footnotetext{
* Corresponding author. Tel./ fax: +86-451-86402590

E-mail address: dongjunwang@hit.edu.cn (Dongjun Wang)
} 
microstructural manipulation, e.g. preparation of homogeneous microstructure and elimination of casting defects.

In recent years, TiAl-based alloys billets are usually produced by traditional ingot metallurgy (IM) $[5,6]$ or powder metallurgy (PM) [7, 8], Nevertheless, the billets are usually forced to be treated by hot isostatic processing (HIP) to eliminate porosity before TMT, which in turn gives rise to longer manufacturing duration and thus higher cost of investment. On the other hand, the cold crucible continuous casting technique has been developed as a novel and effective method to fabricate titanium and its alloys without contamination and defects due to the adoption of electromagnetic cold crucibles (EMCC) [9-11]. Although many attempts have been made to investigate the hot deformation behaviors of conventional as-cast or wrought TiAl-based alloys, few studies are focused on the TMT of titanium aluminide alloy using billets prepared by continuous casting. Thus, based on the advantage of TMT and cold crucible continuous casting technique, it is expected that TiAl-based components with excellent properties can be fabricated by the cold crucible continuous casting combined with TMT process, which can help to understand the underlying relationships between hot deformability and microstructure better, and further accelerate the application of this kind of advanced alloy.

In the present study, the hot deformation behavior of fully lamellar TiAl-based alloy prepared by cold crucible continuous casting was explored in detail. The constitutive equation and processing map were developed based on the flow curves. Then the range of temperatures and strain rates being suitable for successful hot working was determined. The dependence of strain rate and temperature on the evolution of the microstructure was characterized and also discussed.

\section{Material and experimental}

The alloy used in this work was received as a vacuum induction melted Ti-43AI-2Si billet and then was remelted by cold crucible continuous casting under the pulling velocity of $10 \mu \mathrm{m} / \mathrm{s}$ and input power of $55 \mathrm{~kW}$. Cylinder specimens for hot compression test with the aspect ratio of 1.5 (i.e., $9 \mathrm{~mm}$ in height and $6 \mathrm{~mm}$ in diameter) were machined from the ingot with their axes parallel to the solidification direction. Hot compression tests were performed on Gleeble 1500D instrument and true stress-strain data were obtained by an automatic data acquisition system. The specimens were pre-heated at $10^{\circ} \mathrm{Cs}^{-1}$ to the deformation temperature and $1 \mathrm{~min}$ soaking time was kept for equilibrium of the temperature in the sample before compression. Tantalum sheets were placed between specimens and punches to reduce the friction. The deformation temperatures were chosen to be in the range of $1100-1250^{\circ} \mathrm{C}$ with an interval of $50^{\circ} \mathrm{C}$ under the true strain of -0.8 . Since the deformation of gamma-TiAl alloy is very sensitive to the strain rate, the strain 
rates of $10^{-3}, 10^{-2}, 10^{-1}$ and $1 \mathrm{~s}^{-1}$ were selected in this study in order to avoid catastrophic crushing when the strain rate was higher than $1 \mathrm{~s}^{-1}$. And then the specimens were immediately cooled by water quenching after hot deformation.

The specimens were sectioned parallel to the compression axis and mechanically ground, then polished by electrolytic method in a solution composed of $60 \mathrm{vol} \%$ methanol, $34 \mathrm{vol} \%$ butyl alcohol and $6 \mathrm{vol} \%$ perchloric acid at $30 \mathrm{~V}$ and $-25^{\circ} \mathrm{C}$. The etched reagent was the Kroll's reagent, which was $50 \mathrm{vol} \%$ nitric acid, 3 vol\% hydrofluoric acid and 92vol\% purified water. The microstructure was observed by a Leica DMI5000M optical microscope, a Quanta 200FEG electron scanning electron microscope (SEM) in BSE, energy dispersive X-ray analysis (EDX) and EBSD mode.

\section{Results and discussion}

\subsection{Original structure}

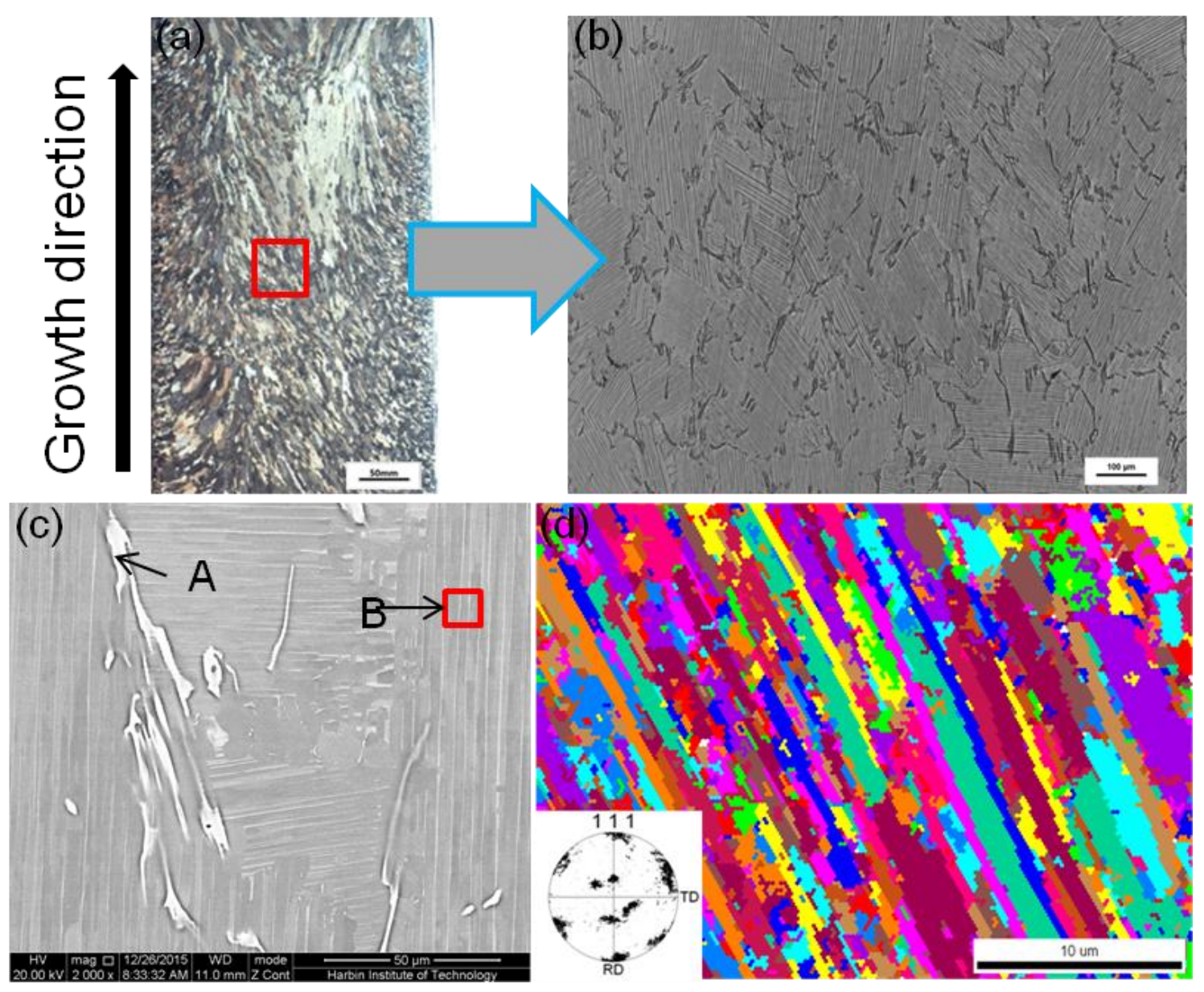

Fig.1. Macrostructure and microstructure of the ingot: (a) low magnification, (b) high magnification by optical microscope, (c) microstructure by SEM-BSE, (d) unique grain color figure and pole figure by EBSD.

The original macrostructure of Ti-43Al-2Si alloy prepared by cold crucible continuous casting is shown in

Fig. 1(a). It can be seen that the macrostructure of the initial material is mainly as-cast equiaxed crystalline 
grains. Fig. 1(b) and 1(c) show the microstructure by SEM-BSE and the chemical composition of the phases are give in Table 1 using EDX analysis. The phases with bright contrast along the boundaries of lamellar colonies are $\xi-\mathrm{Ti}_{5} \mathrm{Si}_{3}$ silicides (as indicated by arrow A) and the lamellar morphologies (alternately gray and white lamellas as indicated by arrow B) are $\alpha_{2}\left(\mathrm{Ti}_{3} \mathrm{Al}\right) / \gamma(\mathrm{TiAl})$ phases as documented in Ref. [12]. The average size of lamellar colonies is measured to be $\sim 100 \mu \mathrm{m}$. In addition, the colonies have different orientations with respect to the surrounding lamellae. The $\gamma$ and $\alpha_{2}$ phase maintain the Blackburnorientation relationship of $(0001)_{\alpha 2} \|\{111\}_{\gamma}$ and $\left.<11 \overline{2} 0>_{\alpha 2} \|<1 \overline{1} 0\right]_{\gamma}$. Fig.1 (d) presents the unique grain color figure (UGCF) and pole figure of original structure. The concentrated distributions in the pole figure illustrate the remarkably texture.

Table 1 The chemical compositions of different phases of Ti-43Al-2Si alloy ingot (at.\%)

\begin{tabular}{cccc}
\hline Position & $\mathrm{Ti}$ & $\mathrm{Al}$ & $\mathrm{Si}$ \\
\hline $\mathrm{A}$ & 59.95 & 10.71 & 29.34 \\
$\mathrm{~B}$ & 51.75 & 47.55 & 0.71 \\
\hline
\end{tabular}

\subsection{Stress-strain curve}

Fig. 2 shows the typical true stress-true strain cures of the Ti-43Al-2Si alloy prepared by cold crucible continuous casting at elevated temperatures. The effects of temperature, strain rate and stain on high temperature flow stress are recorded during the isothermal compression. It can be seen that the stress corresponding to identical strain increases sharply with the decreasing of deformation temperatures and the increasing of strain rates. Since the alloy deforms in different phase regions from $\gamma+\alpha_{2}\left(1100^{\circ} \mathrm{C}\right)$ to $\gamma+\alpha$ $\left(1250^{\circ} \mathrm{C}\right)$, the deformation temperature is a critical factor for the flow stress. One can see that the true stresstrue strain curves fluctuate slightly at low temperatures $\left(1100-1150^{\circ} \mathrm{C}\right)$ with relatively high strain rates $(0.1-$ $1 \mathrm{~s}^{-1}$ ) due to the flow instability and propagation of cracks. The high strain rates give rise to fast deformation of the alloys, which leads to insufficient dynamic softening and contributes to severer work hardening. Furthermore, the flow stress increases greatly to peak value caused by work hardening at the initial stage of deformation and then continuous flow softening occurs for dynamic recovery (DRY) and dynamic recrystallization (DRX) [13]. With further increasing of the strain, the stress remains a stable state, resulting from the synergetic effect of work hardening and dynamic softening. In particular, the strength of the TiAlbase alloy is higher in this work when compared with the result of Ref. [14] under the same test conditions, which exhibits good high-temperature properties of alloys prepared by cold crucible continuous casting. 
Beside the reinforced effect of smaller grains of the present study, it is also believed that the higher volume fraction of grain boundaries $(\mathrm{GB})$ can act as strong obstacles to the dislocation motion, which will accommodate more deformation energy and lead to higher strength.
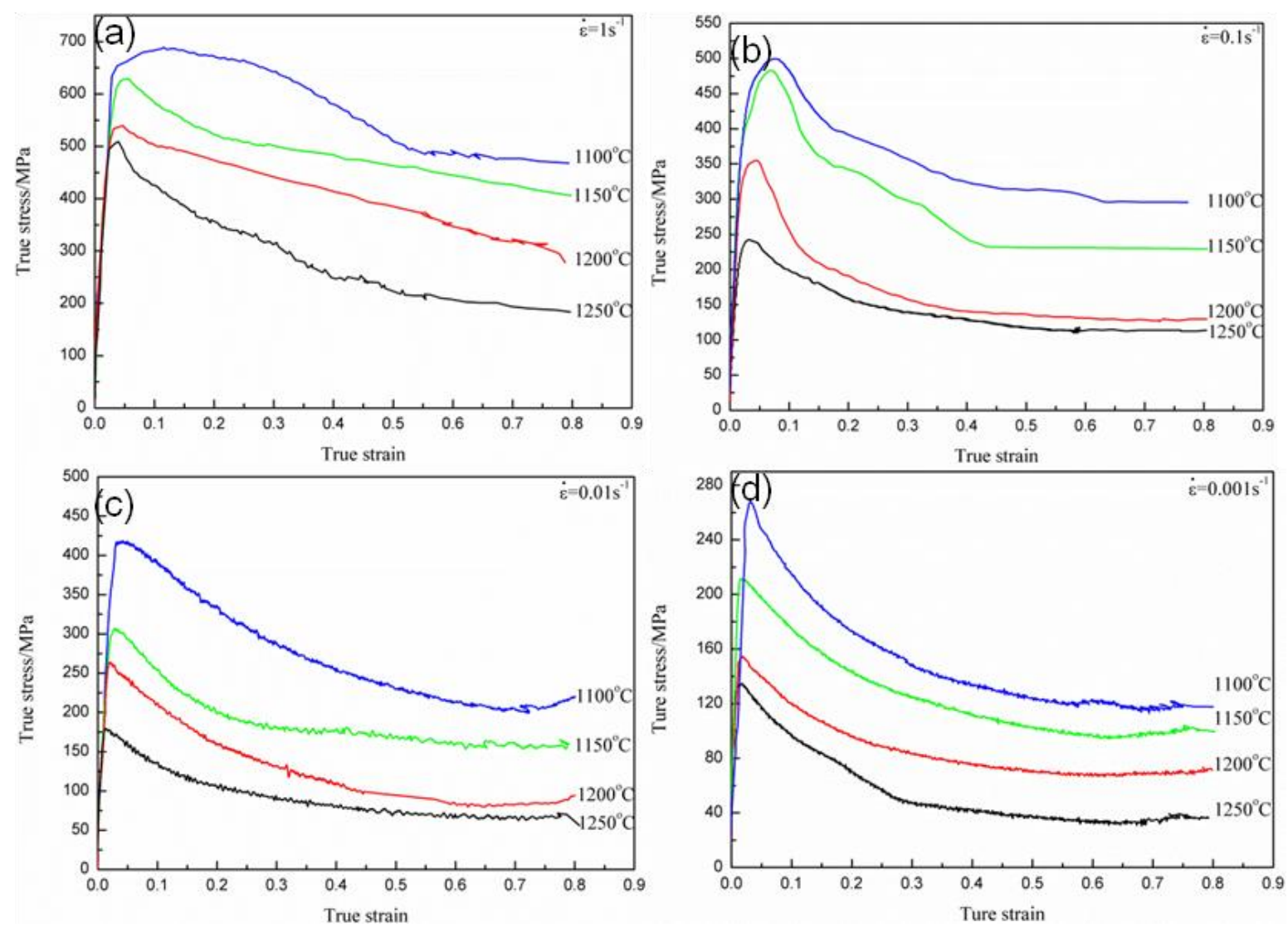

Fig.2. Flow curves of the Ti-43Al-2Si alloy under different deformation conditions: (a) $1 \mathrm{~s}^{-1}$, (b) $0.1 \mathrm{~s}^{-1}$, (c) $0.01 \mathrm{~s}^{-1}$, (d) $0.001 \mathrm{~s}^{-1}$.

\subsection{Constitutive equation}

In order to better understand the underlying mechanism of deformation at elevated temperature, the relationships between flow behavior of the material and deformation parameters can be expressed as following equations $[6,15,16]$ :

$$
\begin{aligned}
& \dot{\varepsilon}=A_{1} \sigma^{n_{1}} \exp [-Q /(R T)] \quad(\alpha \sigma<0.8) \\
& \dot{\varepsilon}=A_{2} \exp (\beta \sigma) \exp [-Q /(R T)] \quad(\alpha \sigma<0.8) \\
& \dot{\varepsilon}=\mathrm{A}[\sinh (\alpha \sigma)]^{n} \exp [-\mathrm{Q} /(\mathrm{R} T)] \quad(\text { for all) } \\
& Z=\dot{\varepsilon} \exp [\mathrm{Q} /(\mathrm{R} T)]=\mathrm{A}[\sinh (\alpha \sigma)]^{n} \\
& \alpha=\beta / n_{1}
\end{aligned}
$$

Where $\mathrm{A}_{1}, \mathrm{~A}_{2}, \mathrm{~A}, \mathrm{n}_{1}, \beta$ and $\alpha$ are the material constants, $\mathrm{R}$ is the Boltzmann constant $\left(8.31 \mathrm{~J} \cdot(\mathrm{mol} \cdot \mathrm{K})^{-1}\right)$, $T$ is the deformation temperature $(\mathrm{K}), n$ is the stress exponent, $\mathrm{Q}$ is the activation energy of deformation 
$\left(\mathrm{kJ} \cdot \mathrm{mol}^{-1}\right), \sigma$ is the flow stress $(\mathrm{MPa}), \dot{\varepsilon}$ is the strain rate $\left(\mathrm{s}^{-1}\right), \mathrm{Z}$ is the parameter of Zener-Hollomon. By making the transformation of natural logarithm for Eqs.(1)-(3), the parameter $\mathrm{n}_{1}, \beta, \mathrm{n}$ and $\mathrm{Q}$ can be written as follows:

$$
\begin{aligned}
& \mathrm{n}_{1}=\left(\frac{\partial \ln \dot{\varepsilon}}{\partial \ln \sigma}\right)_{T} \\
& \beta=\left(\frac{\partial \ln \dot{\varepsilon}}{\partial \sigma}\right)_{T} \\
& \mathrm{n}=\left[\frac{\partial \ln \dot{\varepsilon}}{\partial \ln (\sinh (\alpha \sigma))}\right]_{T} \\
& \mathrm{Q}=\operatorname{Rn} b=\operatorname{Rn}\left[\frac{\partial \ln (\sinh (\alpha \sigma))}{\partial(1 / \mathrm{T})}\right]_{T}
\end{aligned}
$$

The values of these parameter can be obtained from slopes of $\ln \dot{\varepsilon}-\ln \sigma_{\mathrm{p}}, \ln \dot{\varepsilon}-\sigma_{\mathrm{p}}, \ln \left[\sinh \left(\alpha \sigma_{\mathrm{p}}\right)\right]-1000 / \mathrm{T}$ and $\ln \left[\sinh \left(\alpha \sigma_{\mathrm{p}}\right)\right]-\ln \dot{\varepsilon}$ in Fig. 3, according to Eqs. (6)-(9). Especially, it is important to note that the deformation activation energy is influenced by DRX and the test conditions, which is shown in Fig. 4. As can be seen, the 3D deformation activation energy map having saddle-like shape varies from 360 to $537 \mathrm{~kJ} \cdot \mathrm{mol}^{-1}$ with an average value of $451.15 \mathrm{~kJ} \cdot \mathrm{mol}^{-1}$. Based on this map, it is appropriate for the alloys to be hot processed in the zone with stable and relatively smaller $Q$ values. For the Ti-43Al-2Si alloy prepared by cold crucible continuous casting in this study, the optimal hot working conditions can be determined at the temperatures between $1180^{\circ} \mathrm{C}$ and $1230^{\circ} \mathrm{C}$ using low strain rate with $\mathrm{Q}$ values in a special range of 410 $460 \mathrm{~kJ} \cdot \mathrm{mol}^{-1}$. The other zone with $\mathrm{Q}$ values of $360-460 \mathrm{~kJ} \cdot \mathrm{mol}^{-1}$ is instability in the processing map, which will be discussed in the following section.
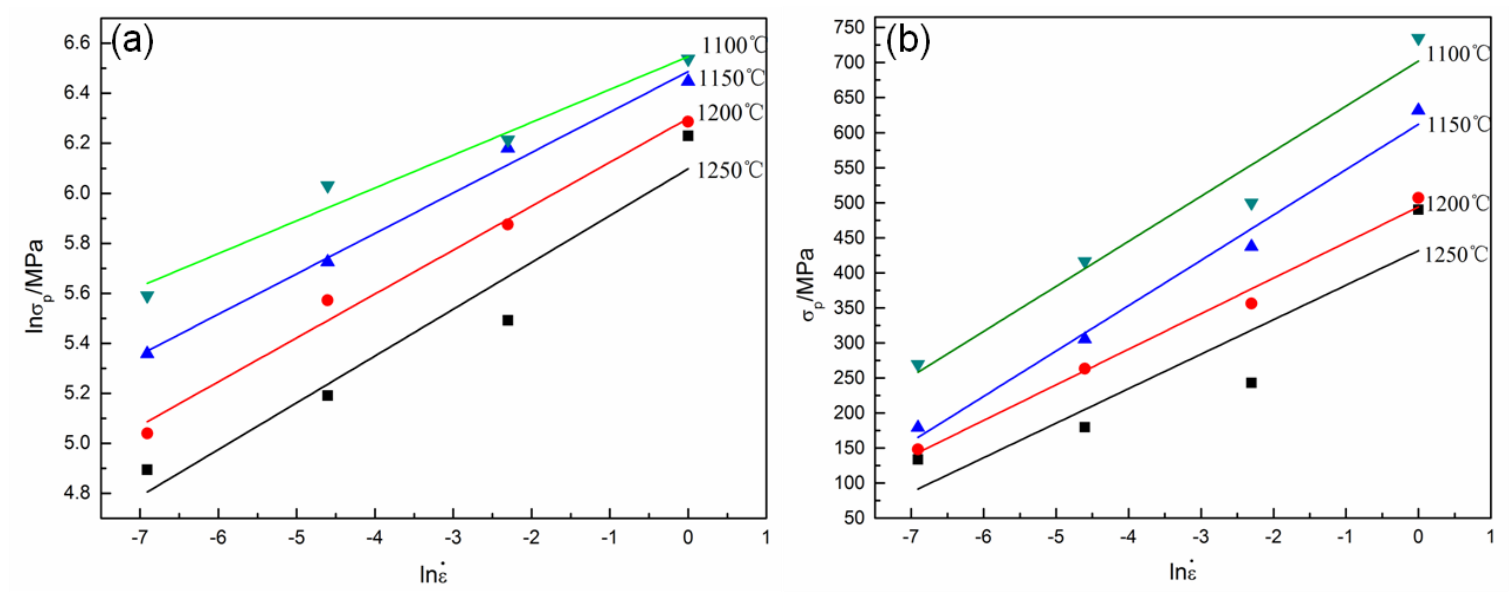

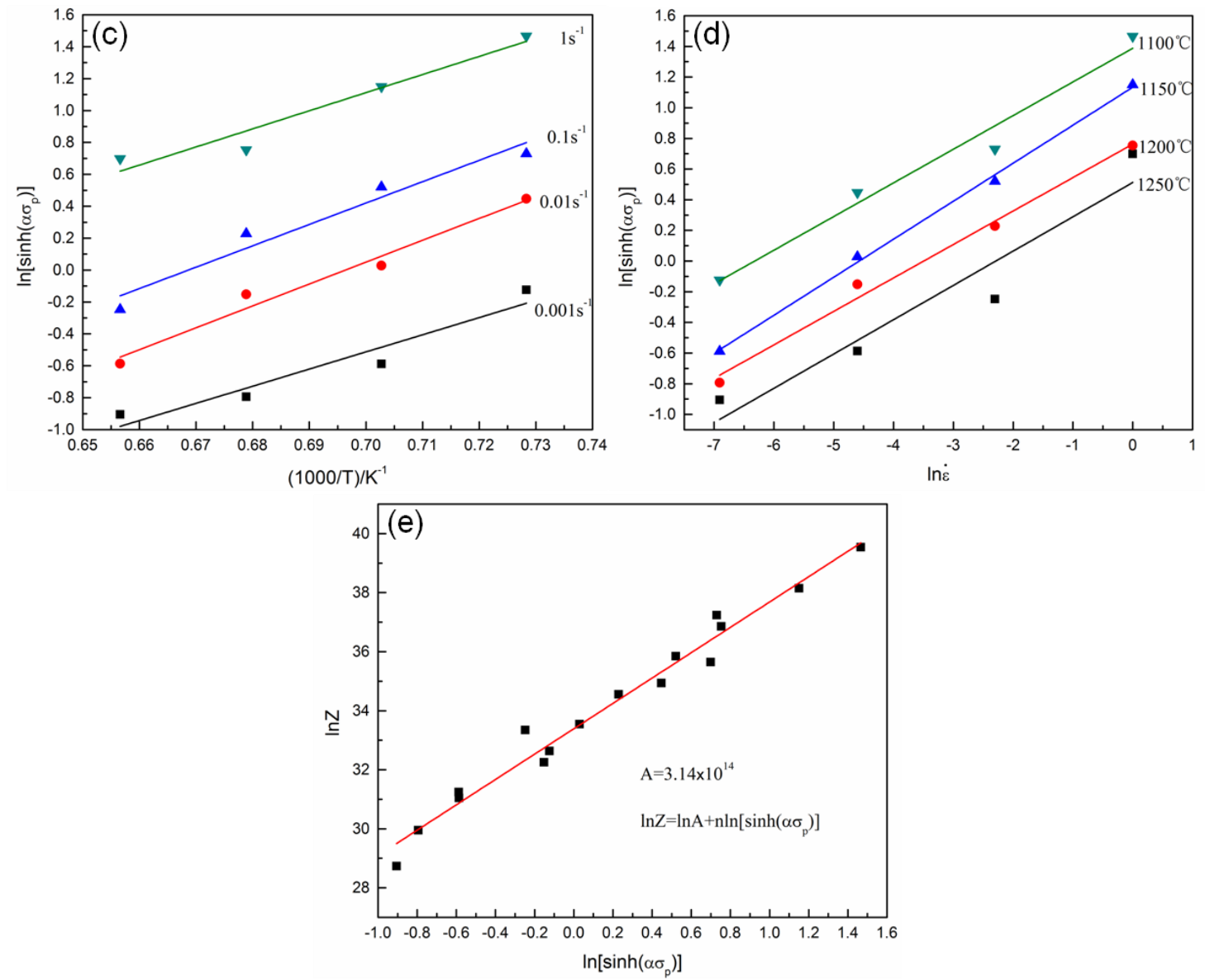

Fig. 3. The linear relationship: (a) $\ln \dot{\varepsilon}-\ln \sigma_{\mathrm{p}}$, (b) $\ln \dot{\varepsilon}-\sigma_{\mathrm{p}}$, (c) $\ln \left[\sinh \left(\alpha \sigma_{\mathrm{p}}\right)\right]-1000 / \mathrm{T}$, (d) $\ln \left[\sinh \left(\alpha \sigma_{\mathrm{p}}\right)\right]-\ln \dot{\varepsilon}$, (e) $\ln \mathrm{Z}-\ln \left[\sinh \left(\alpha \sigma_{\mathrm{p}}\right)\right]$.

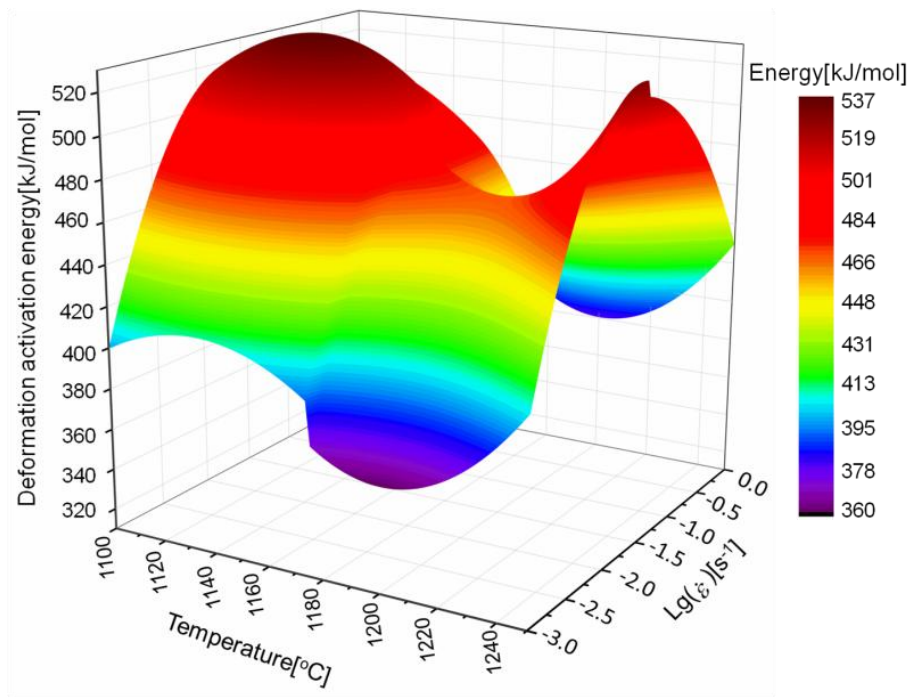

Fig. 4. The 3D deformation activation energy map.

By taking account the average value of $Q$ into Eq. (4), the following equation can be obtained:

$$
Z=\dot{\varepsilon} \exp [451145 /(\mathrm{R} T)]=\mathrm{A}[\sinh (\alpha \sigma)]^{n}
$$

For convenience of discussion, the Zener-Hollomon parameter (Z), which can give the relationship between deformation temperature and strain rate [17], is utilized to investigate the working processes. The 
detailed discussions of the deformation condition, the constitutive relationships and the microstructural evolutions during high temperature deformation will be given later in this article.

Based on the relationship of $\ln \mathrm{Z}-\ln \left[\sinh \left(\alpha \sigma_{\mathrm{p}}\right)\right]$ (Fig. 3(e)), by making the transformation of natural logarithm for Eq. (10), the value $\ln A$ can be acquired from the intercept of relationship of $\ln Z-\ln \left[\sinh \left(\alpha \sigma_{p}\right)\right]$ as shown in Table 2. Finally, the constitutive equation and flow stress equation at elevated temperatures $\left(1100-1250^{\circ} \mathrm{C}\right)$ for this Ti-43Al-2Si alloy can be derived as followings:

$$
\begin{aligned}
\dot{\varepsilon} & =3.14 \times 10^{14}[\sinh (0.002861 \sigma)]^{4.293} \exp [-451145 /(R T)] \\
\sigma_{\mathrm{p}} & =338.41 \ln \left\{\left(\mathrm{z} /\left(3.14 \times 10^{14}\right)\right)^{1 / 4.293}+\left[\left(\mathrm{z} /\left(3.14 \times 10^{14}\right)\right)^{2 / 4.293}+1\right]^{1 / 2}\right\}
\end{aligned}
$$

Table 2 Calculation results of deformation activation energy and material parameter

\begin{tabular}{cccc}
\hline$\alpha$ & $n$ & $Q_{\text {ave }} / \mathrm{kJ} / \mathrm{mol}$ & $\mathrm{A}$ \\
\hline 0.002861 & 4.293 & 451.1452 & $3.14 \times 10^{14}$
\end{tabular}

Furthermore, the theoretical values of peak stress have been calculated by the constitutive equation at different deformation temperature and strain rates. Comparing the theoretical values with experimental results, the results are shown in Fig.5. As can be seen, there exists a linear relationship between these two values with correlation coefficient of 0.975 , which reveals that it is reliable to use this constitutive equation to describe the hot deformation process of this alloy.

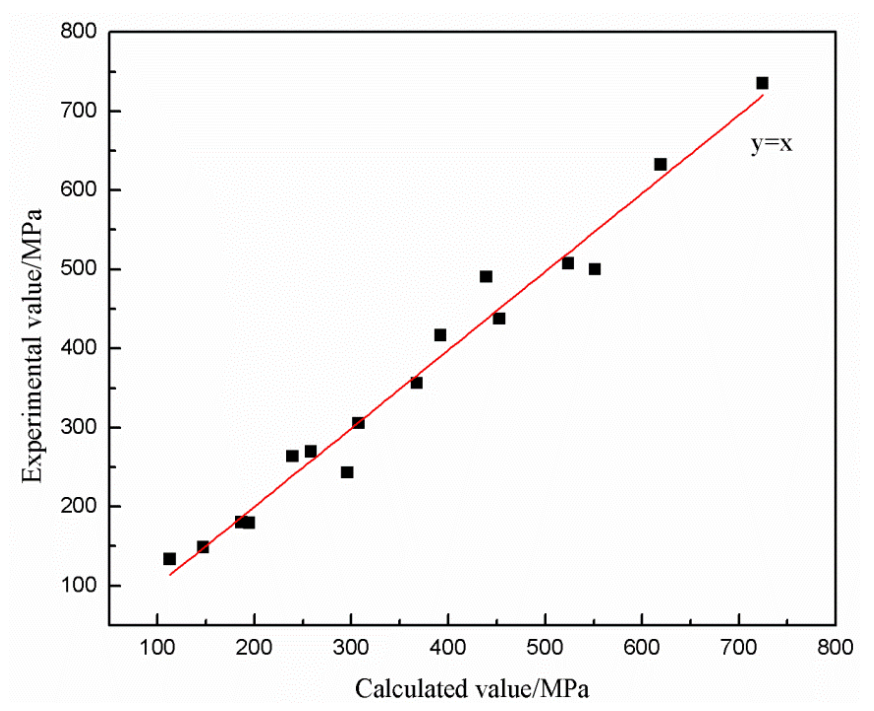

Fig. 5. The comparison of peak stress values from experiment and calculation.

\subsection{D processing map}

In order to obtain the optimal deformation parameters during hot working, the processing map is a direct method for this investigation. The material processing is considered to be a system of power dissipation 
based on dynamic material model (DMM) suggested by Prasad et al [5, 18-20]. The power dissipation, which occurs during the deformation, includes two aspects: (1) the forming of heat and (2) the causing of microstructural evolution. The efficiency of power dissipation of the alloy can be presented by a dimensionless parameter and is given by:

$$
\eta=\frac{J}{J_{\max }}=\frac{2 m}{m+1}
$$

Where $m$ is strain rate sensitivity, which relates to the proportion of the power dissipation. $J$ is the cocontent and is related the power dissipated by DRY, DRX, phase transformation, internal and wedge crack. $J_{\max }$ is the maximum possible dissipation. According to the equation, the variation of $\eta$ with temperature and strain rate constitutes the power dissipation map. Although, it is usually optimized for the deformation to be performed in high efficiency domains, some other instable factors can also cause high efficiency of power dissipation. Thus, it is necessary to develop an instable map for this alloy based on the principle of maximum rate of entropy production as $[7,21]$ :

$$
\xi(\dot{\varepsilon})=\frac{\partial \ln \left(\frac{m}{m+1}\right)}{\partial \ln \dot{\varepsilon}}+m
$$

Where $\xi(\dot{\varepsilon})$ is the instability parameter. Plotting the instability parameter $\xi(\dot{\varepsilon})$ with respect to the deformation temperature and strain rate gives an instable map. It is also of importance to note that the flow stabilities happen in the regions with negative $\xi(\dot{\varepsilon})$ values. By overlapping the power dissipation map with instable map, the 2D processing map of Ti-43Al-2Si alloy can be developed with certain strain of 0.8 as shown in Fig. 6.

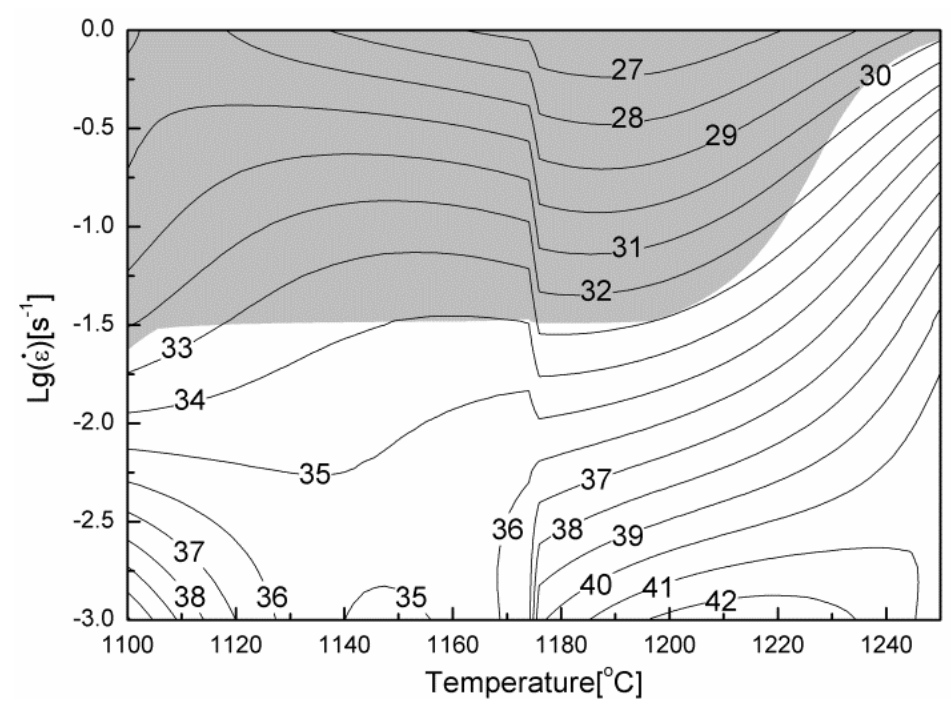

Fig. 6. The 2D processing map of Ti-43Al-2Si alloy prepared by cold crucible continuous casting with at true strain of 0.8 (the shadow indicates the instability domain). 
Fig. 6 exhibits that the peak efficiency of power dissipation is calculated to be $42 \%$ and the deformation occurs in the temperature range of $1195-1235^{\circ} \mathrm{C}$ with the strain rate of $0.001 \mathrm{~s}^{-1}$. Moreover, one can see that it is not realizable to hot working of this alloy at low temperature or with high strain rate as the efficiency of power dissipation is low and the instability parameter is negative. In this case, the movement of dislocations is not sufficient and thus the dislocation pile-up easily takes place. The broken line is found around $1175^{\circ} \mathrm{C}$, which is the phase transformation $\left(\gamma+\alpha_{2} \rightarrow \gamma+\alpha\right)$ temperature for Ti-43AI-2Si alloy. This efficiency inflection feature caused by the eutectoid reaction has also been reported in the Ref. [22]. Base on the processing map, the optimal hot working window for the present alloy is at the temperatures ranging from $1150^{\circ} \mathrm{C}$ to $1250^{\circ} \mathrm{C}$ using strain rate lower than $0.01 \mathrm{~s}^{-1}$ with true strain of 0.8 .

Table 3 The samples deformed under different processing condition

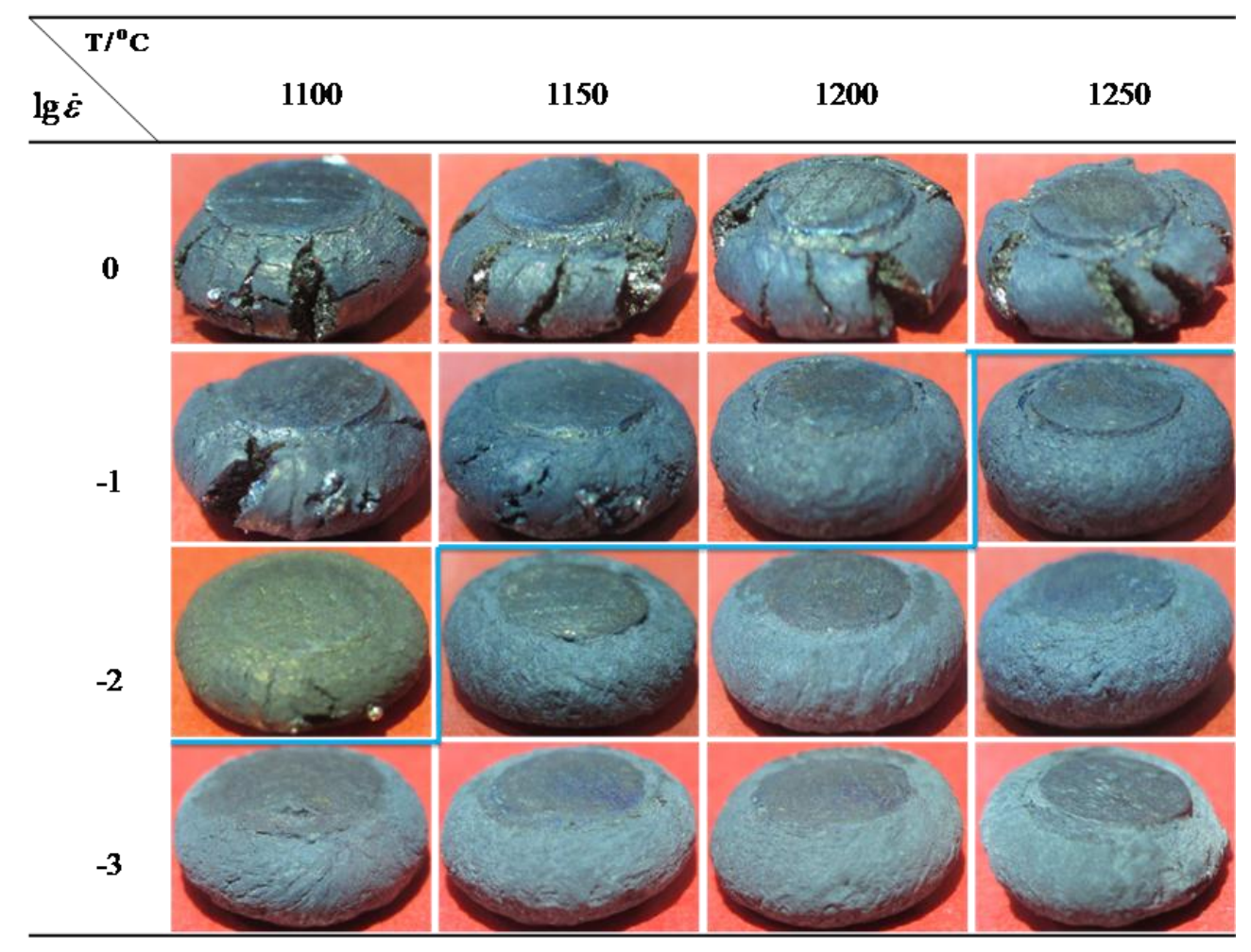

Table 3 shows the appearance of deformed samples at different processing conditions. It can be observed that the samples become fracture by shear failure with crack angle of $90^{\circ}$ or $45^{\circ}$ on the surfaces of deformed samples. When the strain rate is $1 \mathrm{~s}^{-1}$, all the samples fracture with evident cracks on the surface. In addition, there are no cracks seen for strain rate of $0.1 \mathrm{~s}^{-1}$ at temperatures above $1200^{\circ} \mathrm{C}$, whereas the cracks can also be found at lower deformation temperatures. As shown for the well-formed shape of these samples, the hot deformation of this alloy should be performed using strain rates below $0.01 \mathrm{~s}^{-1}$, which can also be confirmed and predicted by the processing map. 
Fig. 7 presents a schematic illustration of the stress state for the deformed sample. As can be seen, the sample includes three distinct regions according to the deformation degree and the stress state. For the region near the pressure head (region I), the true strain during hot compression is small due to temperature drop and friction, as shown in Fig.7. In addition, region II deforms severely and thus accommodates large strain because of relatively fewer frictions in this region. The region III is the part of bulging resulted from the tensile stress in the tangential direction. The tensile stress in region III becomes greater from the inner part of the sample to the surface. As a result of brittleness for TiAl-based alloys, the fracturing occurs on their surfaces. In order to avoid or decrease tangential tensile tress during deformation, the canned forging has been applied by Niu et al. [23, 24], which is also helpful for homogeneous DRX.

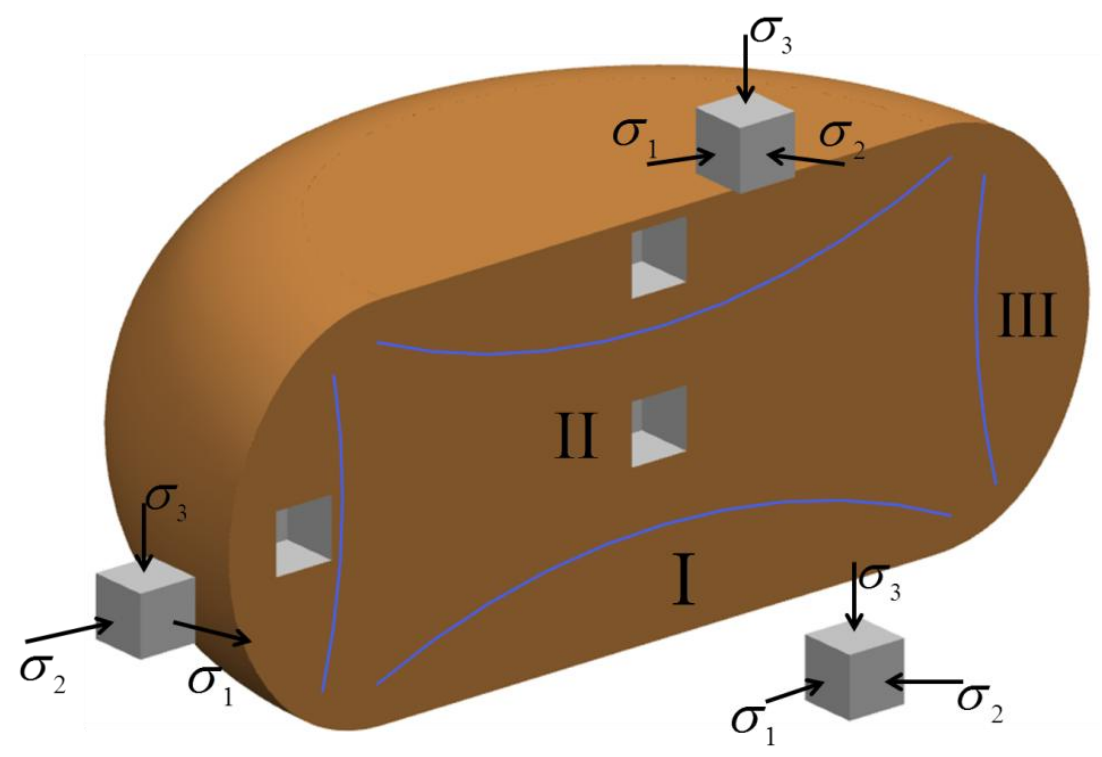

Fig. 7 Stress state of deformed sample

In addition to deformation temperature and strain rate, it is also well document that the strain plays a key role on the deformation behavior of materials $[18,25,26]$. Thus, the 3D processing map can be presented in Fig. 8. The maximum efficiency of power dissipation is calumniated to be $\sim 38 \%$ at the deformation temperature of $1250^{\circ} \mathrm{C}$ with strain of 0.1 . In case of temperature for $1200^{\circ} \mathrm{C}$ with strain of 0.8 , the maximum efficiency of power dissipation can also be determined as $\sim 42 \%$. It can be seen that the instable domain increases with the increasing of strain. In particular, it is difficult to find out the instable domain when the stain is smaller than 0.4 . For the Ti-43Al-2Si alloy in this study, the flow instability will take place when the strain is larger than 0.4 and the strain rate is higher than $0.1 \mathrm{~s}^{-1}$. 

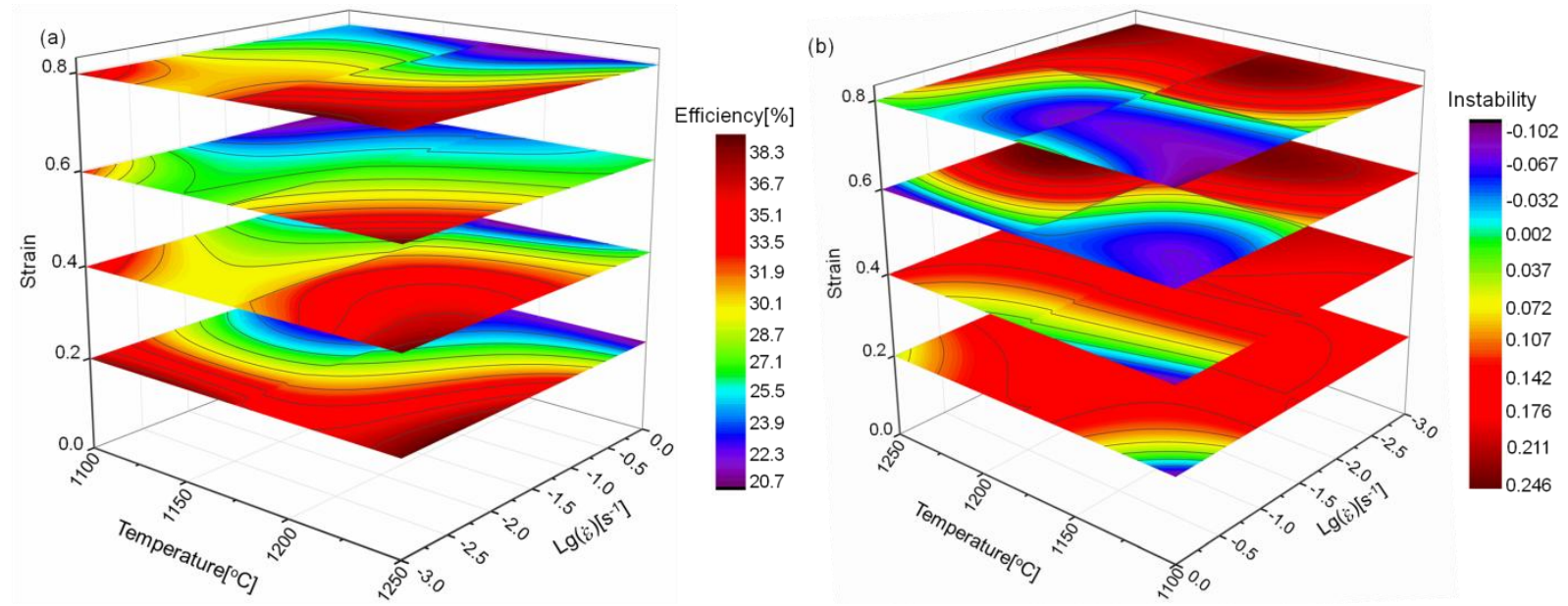

Fig. 8. The 3D processing map: (a) power dissipation map of Ti-43Al-2Si alloy prepared by cold crucible continuous casting, (b) flow instability map of Ti-43Al-2Si alloy prepared by cold crucible continuous casting.

\subsection{Deformation behaviors}

Fig. 9 shows the microstructures in the bulging area (region III) at different deformation conditions. The deformed conditions of sample for $1150^{\circ} \mathrm{C} / 1 \mathrm{~s}^{-1}$ and $1200^{\circ} \mathrm{C} / 0.1 \mathrm{~s}^{-1}$ are in the instable region of the processing map. The macro-sized cracks propagate from surface to the inside along lamellae, as shown in Fig. 9(a), while micro-size pores initiate along the boundaries of $\xi-\mathrm{Ti}_{5} \mathrm{Si}_{3}$ phase (Fig. $\mathbf{9 ( b )}$ ). It can be observed the DRX existing in the microstructure of sample at deformed condition of $1250^{\circ} \mathrm{C} / 0.01 \mathrm{~s}^{-1}$, which is corresponding induced by the high value of power dissipation in processing map. Moreover, the cracks and cavities can be prohibited during deformation of the sample at this appropriate low Z-value condition.

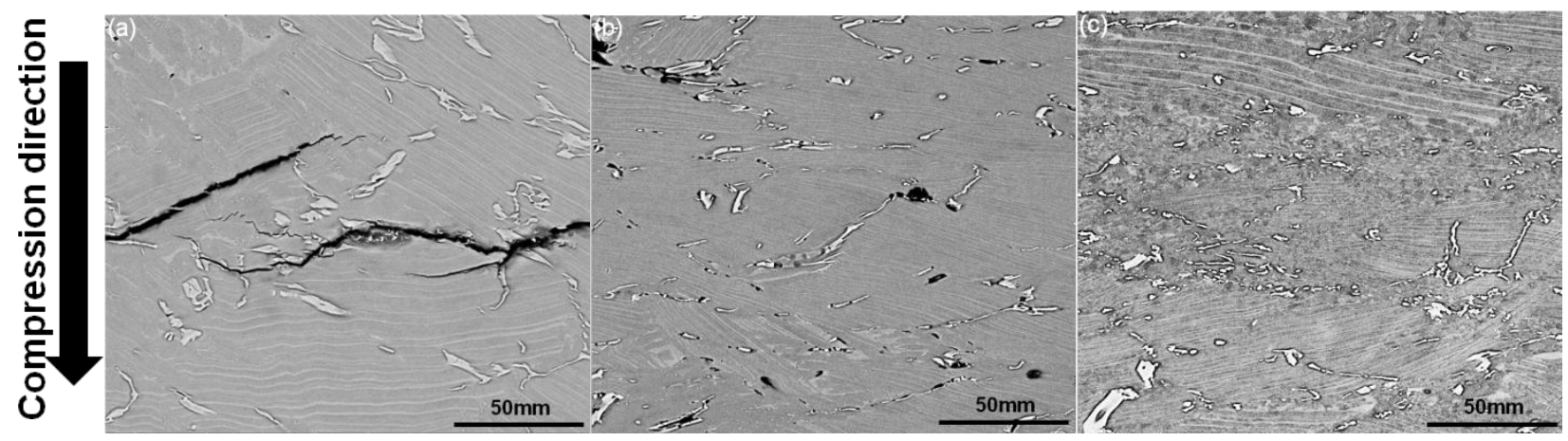

Fig. 9. Microstructure in the region III of Fig. 7 under different deformation conditions: (a) $1150^{\circ} \mathrm{C} / 1 \mathrm{~s}^{-1}$ (InZ=37.91), (b) $1200^{\circ} \mathrm{C} / 0.1 \mathrm{~s}^{-1}$ (InZ=34.32), (c) $1250^{\circ} \mathrm{C} / 0.01 \mathrm{~s}^{-1}$ (InZ=30.83).

The deformed microstructures of samples with large (region II) at different deformation conditions are shown in Fig. 10. It can be seen that the deformation microstructures are obviously different from those of the initial state (Fig. 1). The $\xi-\mathrm{Ti}_{5} \mathrm{Si}_{3}$ phases are crushed and thus distribute along the lamellae or deformation band in chain-like morphology. The lamellae microstructure retains at high $Z$ condition 
$\left(1150^{\circ} \mathrm{C} / 1 \mathrm{~s}^{-1}\right.$, Fig. $\left.10(\mathrm{a})\right)$, whereas the orientation of lamellae turn to the direction which is perpendicular to the compression axis. For lower $Z$ value, the lamellae transform into spheroidized microstructure at the lamellar interfaces or colony boundaries under deformation conditions of $1200^{\circ} \mathrm{C} / 0.1 \mathrm{~s}^{-1} 1$ (InZ=34.32, Fig. 10(b)). The residual lamellae almost disappear and new globularization grains grow up in the lowest $Z$ condition $\left(1250^{\circ} \mathrm{C} / 0.01 \mathrm{~s}-1\right.$, Fig. 10(c)). Compared Figs. 10 (a), (b) and (c), one can see that the deformed microstructure is closely related to the power dissipation efficiency and the DRX will be promoted with the increasing of power dissipation efficiency. In addition, the volume fraction of DRX, which is a regarded as softening mechanism for hot working of full lamellar TiAl-based alloy, will increase with the decreasing of InZ value, as shown below in detail.

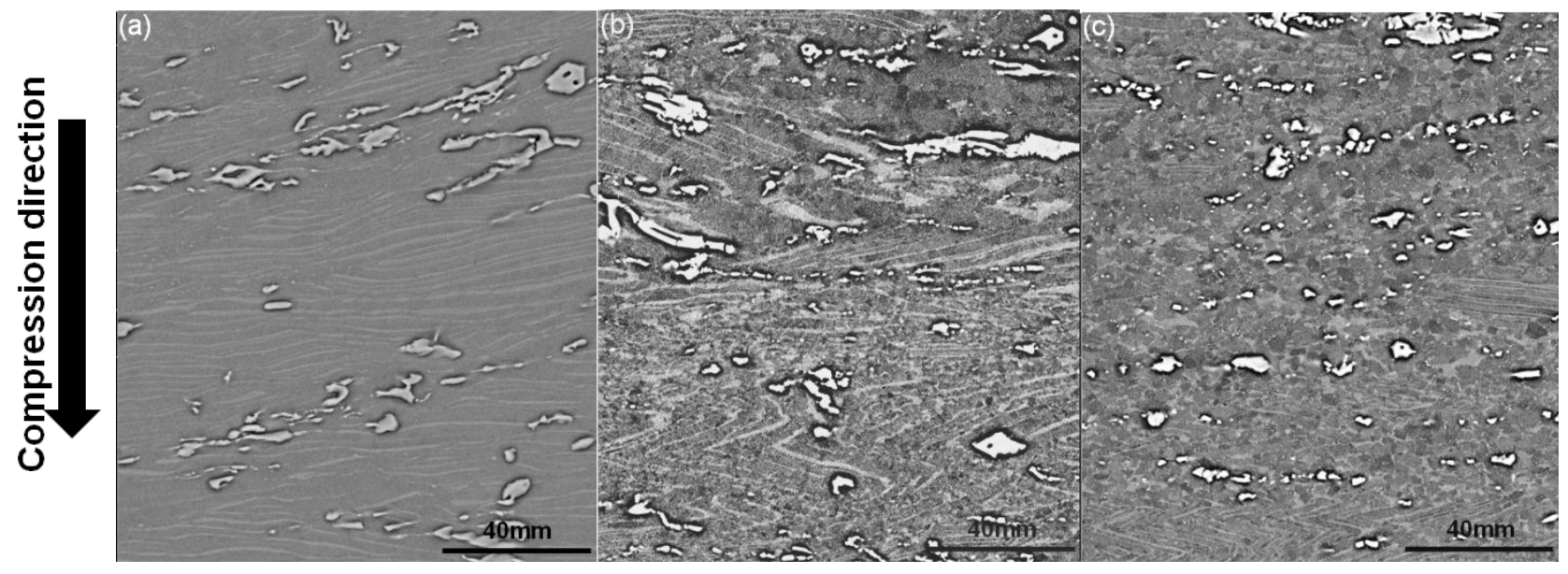

Fig. 10. Microstructure in the region II of Fig. 7 under different deformation conditions: (a) $1150^{\circ} \mathrm{C} / 1 \mathrm{~s}^{-1}$ (InZ=37.91), (b) $1200^{\circ} \mathrm{C} / 0.1 \mathrm{~s}^{-1}(\ln Z=34.32)$, (c) $1250^{\circ} \mathrm{C} / 0.01 \mathrm{~s}^{-1}(\ln Z=30.83)$.

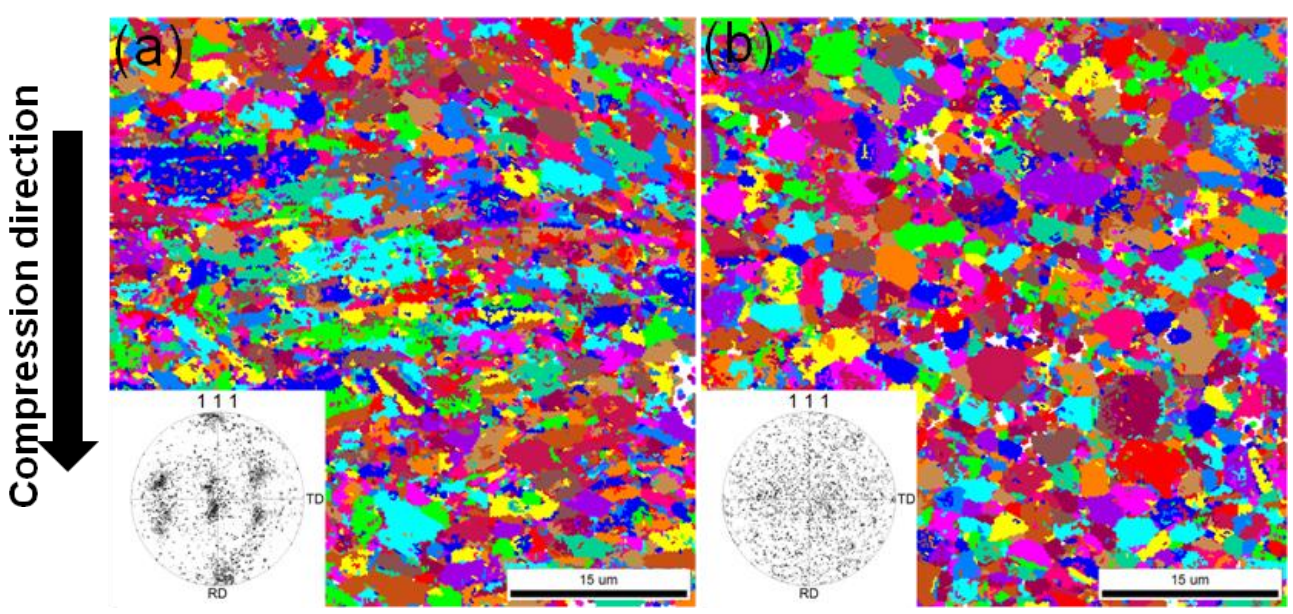

Fig. 11. UGCF and pole figures at strain rate of $0.01 \mathrm{~s}^{-1}$ and different temperature: (a) $1150^{\circ} \mathrm{C}$ (InZ=33.31), (b) $1250^{\circ} \mathrm{C}(\ln Z=30.83)$.

Fig. 11 exhibits UGCF and pole figures obtained by SEM-EBSD mode in the region II of Fig. 7 (similarly hereinafter) at different deformation conditions. It can be noted that all the lamella structures have 
transformed into DRX grains according to the UGCF. A concentrated orientation distribution can also be shown in the pole figure at deformation condition of $1150^{\circ} \mathrm{C} / 0.01 \mathrm{~s}^{-1}$ (inset of Fig. 11(a)), in which the texture is different from that caused by lamella structure in the Fig. 1(d). Furthermore, the grains are slightly flattened in Fig.11 (a) owing to insufficient DRX at this temperature. Compared with Fig. 11(a), the image of Fig. 11(b) shows evident equiaxed fine grains for the condition of $1250^{\circ} \mathrm{C} / 0.01 \mathrm{~s}^{-1}$. In particular, based on the pole figure of Fig. 11(b), a uniform orientation distribution induced by DRX is noted.

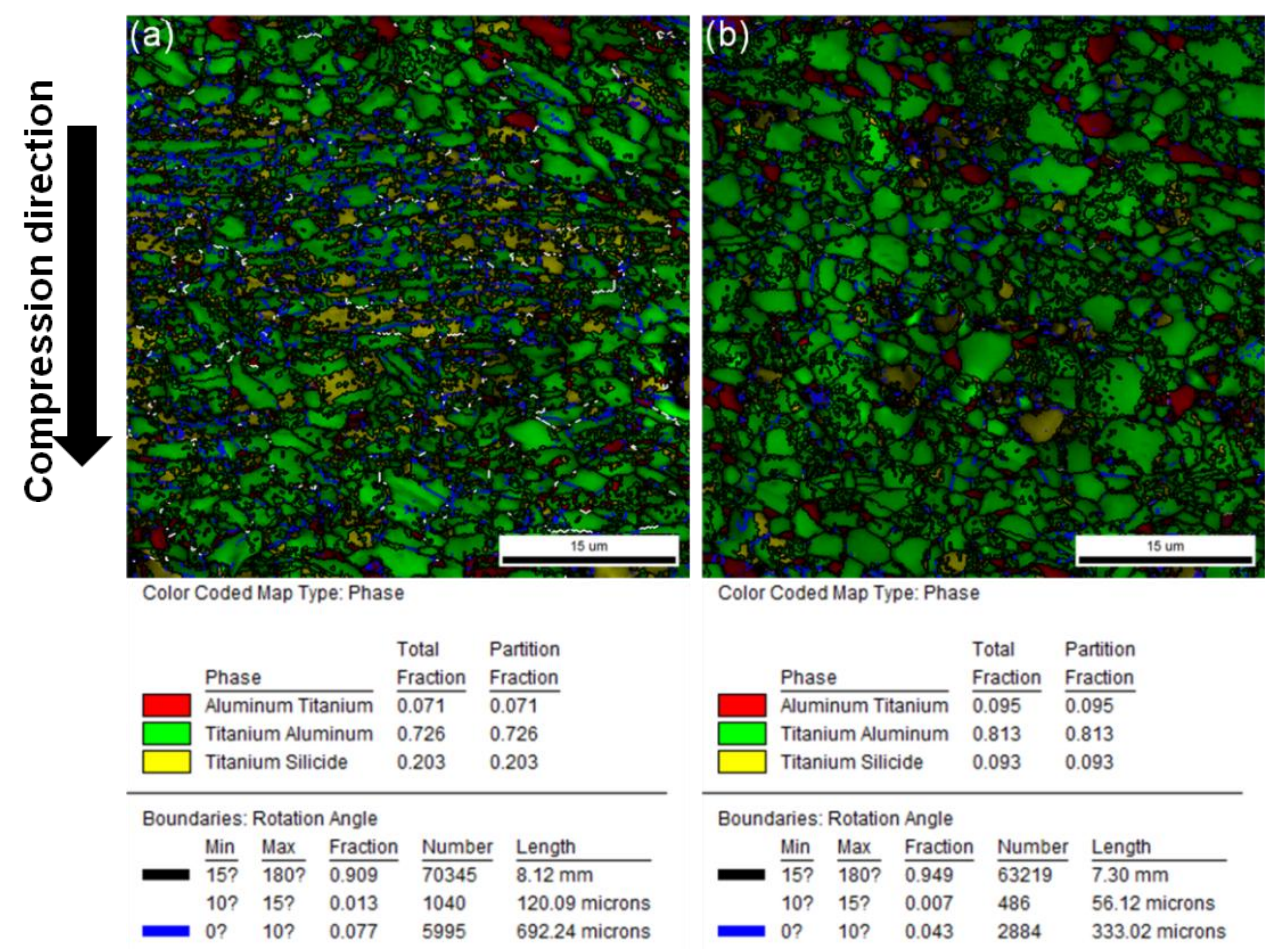

Fig. 12. Phase combined with rotation angle distribution map at strain rate of $0.01 \mathrm{~s}^{-1}$ and different temperature: (a) $1150^{\circ} \mathrm{C}(\ln Z=33.31)$, (b) $1250^{\circ} \mathrm{C}(\ln Z=30.83)$.

Fig.12 shows the phase combined with rotation angle distribution map at different deformation conditions. The $\alpha_{2}\left(\mathrm{Ti}_{3} \mathrm{Al}\right) / \gamma(\mathrm{TiAl})$ phases granulate during the hot deformation process. The fraction of $\xi-\mathrm{Ti}_{5} \mathrm{Si}_{3}$ phases is $\sim 20.3 \%$ at condition of $1150^{\circ} \mathrm{C} / 0.01 \mathrm{~s}^{-1}$ when compared with $9.3 \%$ for the case of $1250^{\circ} \mathrm{C} / 0.01 \mathrm{~s}^{-1}$. The phase transformation $\left(\gamma+\alpha_{2} \rightarrow \gamma+\alpha\right)$ occurs at $1250^{\circ} \mathrm{C}$, thus part of $\xi-\mathrm{Ti}_{5} \mathrm{Si}_{3}$ phases dissolve and more $\alpha$ phases precipitate. Moreover, the low angle grains boundaries (LAGBs, $2^{\circ}-10^{\circ}$ ), medium angle grains boundaries (MAGBs, $5^{\circ}-15^{\circ}$ ) and high angle grain boundaries (HAGBs, $15^{\circ}-180^{\circ}$ ) are also shown by statistical calculation in Fig.12. The fraction of LAGBs at condition of $1150^{\circ} \mathrm{C} / 0.01 \mathrm{~s}^{-1}$ is much higher than that of $1250^{\circ} \mathrm{C} / 0.01 \mathrm{~s}^{-1}$, since the LAGBs are normally the features of substructures formation during isothermal compression [27-29]. The fractions of MAGBs are $1.3 \%$ and $0.7 \%$, respectively, while the fractions of HAGBs are $90.9 \%$ and $94.9 \%$ for these two temperatures. It is well documented that the MAGBs 
are one of the prerequisites for the nucleation of continuous dynamic recrystallization (CDRX), which is associated with the bulging of grain boundaries [30]. On the other hand, another mechanism of DRX is proposed as the discontinue dynamic recrystallization (DDRX), which mainly comprises grain nucleation and growth processes [31,32]. Therefore, due to the low fraction of MAGBs in these samples, the DDRX is deduced as the main DRX mechanism for present alloy in the hot compression.
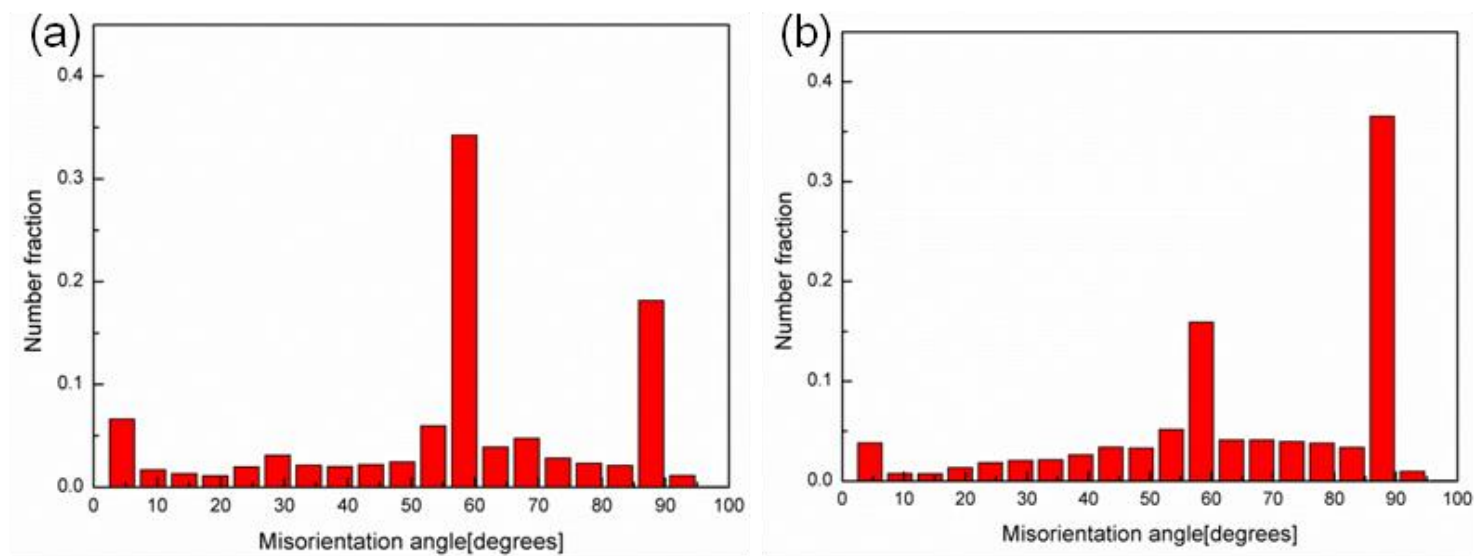

Fig. 13. Misorientation angle distributions of Ti-43Al-2Si alloy at strain rate of $0.01 \mathrm{~s}^{-1}$ and different temperatures: (a) $1150^{\circ} \mathrm{C}(\ln Z=33.31)$, (b) $1250^{\circ} \mathrm{C}(\ln Z=30.83)$.

Fig. 13 shows the misorientation angle distributions at different deformation conditions. The peak value at the deformation condition of $\ln Z=33.31\left(1150^{\circ} \mathrm{C} / 0.01 \mathrm{~s}^{-1}\right)$ is $\sim 60 \pm 3^{\circ}$ for the misorientation angle (Fig. 13(a)), while that shows misorientation angle of $89 \pm 3^{\circ}$ at temperature of $1250^{\circ} \mathrm{C}$ in the Fig. 13 (b). According to the result reported by Jin et al. [33], the misorientation angle of $60 \pm 3^{\circ}$ is closely related to pseudo twin boundaries $60^{\circ}<001>$, which can provide ideal sites and energy for DDRX nucleation and growth and further confirms the result of Fig. 12. In addition, Zong et al. [30] has reported that the misorientation angle of $89 \pm$ $3^{\circ}$ has significant relationship with DDRX nucleation, which makes the grains match and their matrix grains meet the orientation relationship of $\left.89 \pm 3^{\circ}<100\right]$. In contrast to the deformation at high $Z$ value conditions (InZ=33.31, Fig. 13(a)), there exist higher frequency for misorientation angle of $89 \pm 3^{\circ}$ and lower frequency for misorientation angle of $60 \pm 3^{\circ}$ at low $Z$ value conditions $(\ln Z=30.83$, Fig. 13(b)), indicating that the recrystallization process can be improved by the decreasing of $Z$ parameter.

Grain orientation spread (GOS) is the average difference in the orientation between the average grain orientation and the measured grains. GOS map has been used to investigate recrystallization behavior in recent studies [34, 35]. Fig. 14 shows GOS distribution of experimental material with different $Z$ deformation conditions. It can be seen that the DRX and phase transformations mainly occur in the regions with low GOS 
values (blue and green areas). Moreover, the lattice distortion of the sample is severe after deformation at high Z condition (red and orange parts in Fig. 14(a)). It is also of interest to note that relatively more homogeneous grains can be obtained in Fig. 14(b) because of nearly completed DRX after deformation at low $Z$ condition $(\ln Z=30.83)$ in this study.

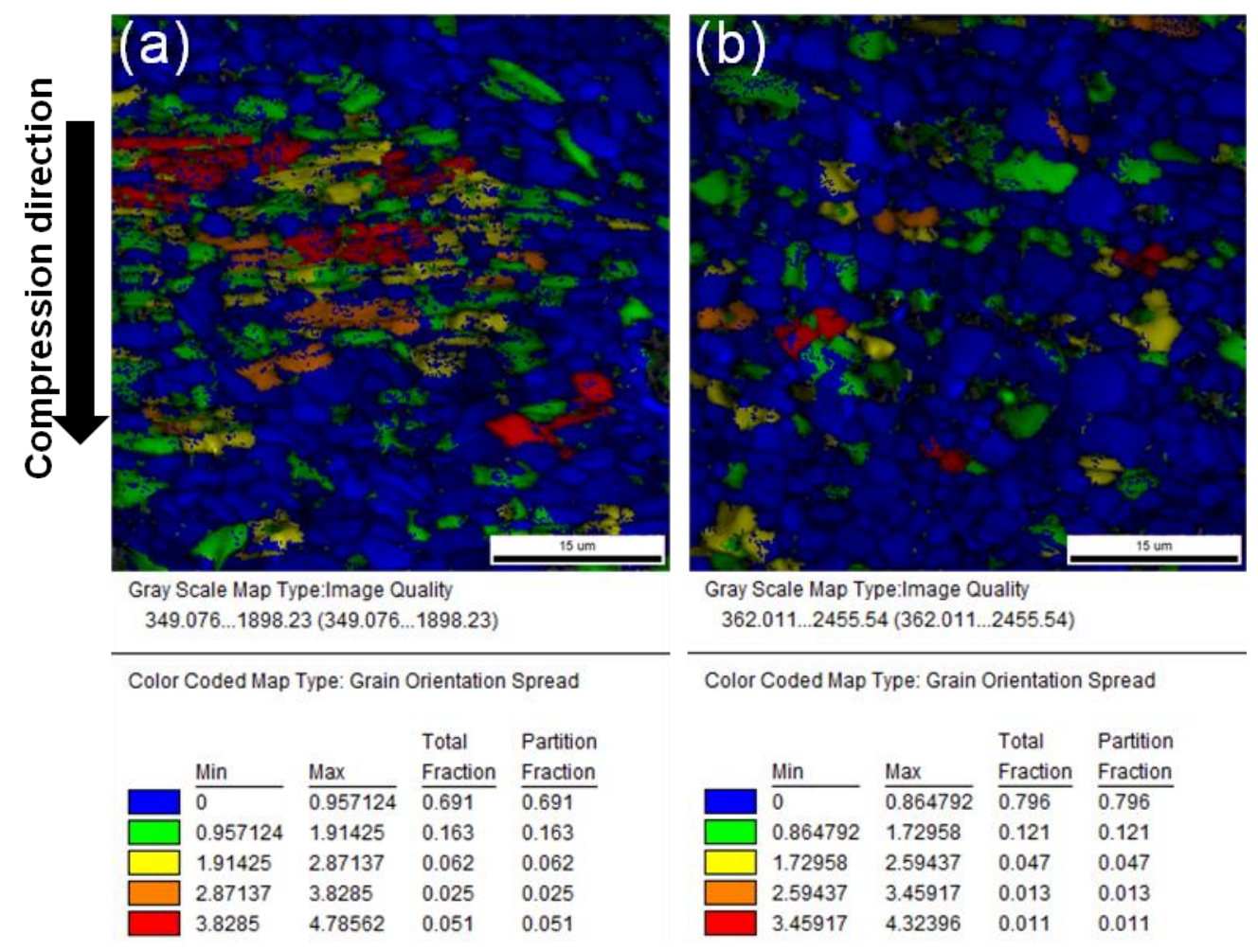

Fig. 14. GOS map of microstructural development at strain rate of $0.01 \mathrm{~s}^{-1}$ and different temperatures: (a) $1150^{\circ} \mathrm{C}(\ln Z=33.31),(b) 1250^{\circ} \mathrm{C}(\ln Z=30.83)$.

\section{Conclusions}

Hot working characterization of cold crucible continuous casting Ti-43Al-2Si alloy was explored by conducting hot compression tests in the temperature range of $1100-1250^{\circ} \mathrm{C}$ using strain rates range of $0.001-1 \mathrm{~s}^{-1}$ in this study. The main conclusions are as follows:

(1) The flow stress is sensitive to the deformation condition, namely deformation temperature and strain rate which is related to the Zener-Hollomom (Z) parameter. The flow stress decreases with the decreasing of $Z$ parameter corresponding to increase of deformation temperature and decrease of strain rate. Moreover, the flow stress appears a peak value in the initial deformation state, and the flow softening occurs with further increasing of strain.

(2) The relationship between flow stress and deformation temperature and strain rate has been established by the constitutive equation and flow stress equation. The deformation parameters can be 
described by Zener-Hollomon parameter. The average deformation activation energy is $451.15 \mathrm{~kJ} / \mathrm{mol}$, which is higher than that for self-diffusion of $\mathrm{Al}$ in $\gamma$ phase.

(3) The deformation activation energy map and processing map of cold crucible continuous casting Ti43Al-2Si alloy has been obtained. It is found that the appropriate hot working domain of this alloy is within the deformation temperature window from $1150^{\circ} \mathrm{C}$ to $1250^{\circ} \mathrm{C}$ and in the strain rate range of $0.001-0.01 \mathrm{~s}^{-1}$ with a strain of 0.8 . Based on microstructural observation, the flow instabilities can be efficiently avoided in the appropriate domain during hot working.

(4) The softening mechanisms of present alloy include dynamic recrystallization, and globularization and reorientation of lamellae. Based on microstructural observation, the main mechanism of DRX is approved to be discontinue dynamic recrystallization and the recrystallization process is promoted with decreasing of $Z$ parameter in this study.

\section{Acknowledgments}

The authors would like to thank the financial support from the Program for Changjiang Scholars and Innovative Research Team in University (No. IRT1229), the Natural Science Foundation of Heilongjiang Province (No.E201425), the Fundamental Research Funds for the Central Universities (No. HIT.KLOF.2013021 and HIT.MKSTISP.2016019), the Postdoctoral Scientific Research Development Fund of Heilongjiang Province (No. LBH-Q15040) and the National Science Foundation of China (No. 51471062).

\section{References:}

[1] I.H. Katzarov, A.T. Paxton, Atomistic studies of interactions between the dominant lattice dislocations and $\mathrm{y} / \mathrm{Y}$ lamellar boundaries in lamellar Y-TiAl, Acta Mater. 57 (2009) 3349-3366.

[2] M.R. Kabir, L. Chernova, M. Bartsch, Numerical investigation of room-temperature deformation behavior of a duplex type YTiAl alloy using a multi-scale modeling approach, Acta Mater. 58 (2010) 5834-5847.

[3] M. Rester, F.D. Fischer, C. Kirchlechner, T. Schmoelzer, H. Clemens, G. Dehm, Deformation mechanisms in micronsized PST TiAl compression samples: Experiment and model, Acta Mater. 59 (2011) 3410-3421.

[4] S.Z. Zhang, C.J. Zhang, Z.X. Du, Z.P. Hou, P. Lin, F.T. Kong, Y.Y. Chen, Deformation behavior of high Nb containing TiAl based alloy in $\alpha+\gamma$ two phase field region, Mater. Des. 90 (2016) 225-229.

[5] F. Yang, F. Kong, Y. Chen, S. Xiao, Hot workability of as-cast Ti-45Al-5.4V-3.6Nb-0.3Y alloy, J. Alloys Compd. 589 (2014) 609-614.

[6] F. Kong, Y. Chen, D. Zhang, S. Zhang, High temperature deformation behavior of Ti-46Al-2Cr-4Nb-0.2Y alloy, Mater. Sci. Eng., A, 539 (2012) 107-114.

[7] Y. Sun, Z. Wan, L. Hu, J. Ren, Characterization of hot processing parameters of powder metallurgy TiAl-based alloy based on the activation energy map and processing map, Mater. Des. 86 (2015) 922-932.

[8] K.P. Rao, Y.V.R.K. Prasad, K. Suresh, Hot working behavior and processing map of a $\mathrm{Y}$-TiAl alloy synthesized by powder metallurgy, Mater. Des. 32 (2011) 4874-4881.

[9] F. Durand, The electromagnetic cold crucible as a tool for melt preparation and continuous casting, Int. J. Cast Met. 
Res. 18 (2015) 93-107.

[10] R. Chen, J. Yang, H. Ding, F. Huang, Y. Su, J. Guo, H. Fu, Magnetic field in a near-rectangular cold crucible designed for continuously melting and directionally solidifying TiAl alloys, J. Mater. Process. Technol. 212 (2012) 19341940.

[11] H. Ding, The mechanism of hot crack formation in Ti-6A1-4V during cold crucible continuous casting, China Foundry, 1 (2004) 28-34.

[12] J. Fan, X. Li, Y. Su, J. Guo, H. Fu, The microstructure parameters and microhardness of directionally solidified Ti43Al-3Si alloy, J. Alloys Compd. 506 (2010) 593-599.

[13] X. Liang, Y. Liu, H. Li, C. Zhou, G. Xu, Constitutive relationship for high temperature deformation of powder metallurgy Ti-47Al-2Cr-2Nb-0.2W alloy, Mater. Des. 37 (2012) 40-47.

[14] S. Dong, R. Chen, J. Guo, H. Ding, Y. Su, H. Fu, Deformation behavior and microstructural evolution of directionally solidified TiAINb-based alloy during thermo-compression at 1373-1573K, Mater. Des. 84 (2015) 118-132.

[15] L. Cheng, H. Chang, B. Tang, H. Kou, J. Li, Deformation and dynamic recrystallization behavior of a high $\mathrm{Nb}$ containing TiAl alloy, J. Alloys Compd. 552 (2013) 363-369.

[16] B. Liu, Y. Liu, W. Zhang, J.S. Huang, Hot deformation behavior of TiAl alloys prepared by blended elemental powders, Intermetallics, 19 (2011) 154-159.

[17] M. Hasegawa, M. Yamamoto, H. Fukutomi, Formation mechanism of texture during dynamic recrystallization in $\gamma^{-}$ TiAl, nickel and copper examined by microstructure observation and grain boundary analysis based on local orientation measurements, Acta Mater. 51 (2003) 3939-3950.

[18] J. Li, J. Liu, Z. Cui, Characterization of hot deformation behavior of extruded ZK60 magnesium alloy using 3D processing maps, Mater. Des. 56 (2014) 889-897.

[19] K.P. Rao, Y.V.R.K. Prasad, Processing map and hot working mechanisms in a P/M TiAl alloy composite with in situ carbide and silicide dispersions, Mater. Sci. Eng., A, 527 (2010) 6589-6595.

[20] Y.V.R.K. Prasad, H.L. Gegel, S.M. Doraivelu, J.C. Malas, J.T. Morgan, K.A. Lark, D.R. Barker, Modeling of dynamic material behavior in hot deformation: Forging of Ti-6242, Metall. Trans. A, 15 (1984) 1883-1892.

[21] J. Liu, Z. Cui, C. Li, Analysis of metal workability by integration of FEM and 3-D processing maps, J. Mater. Process. Technol. 205 (2008) 497-505.

[22] Z. Du, S. Jiang, K. Zhang, The hot deformation behavior and processing map of Ti-47.5Al-Cr-V alloy, Mater. Des. 86 (2015) 464-473.

[23] H.Z. Niu, Y.Y. Chen, Y.S. Zhang, J.W. Lu, W. Zhang, P.X. Zhang, Producing fully-lamellar microstructure for wrought beta-gamma TiAl alloys without single $\alpha$-phase field, Intermetallics, 59 (2015) 87-94.

[24] H.Z. Niu, F.T. Kong, Y.Y. Chen, C.J. Zhang, Low-temperature superplasticity of forged Ti-43Al-4Nb-2Mo-0.5B alloy, J. Alloys Compd. 543 (2012) 19-25.

[25] A. Mohamadizadeh, A. Zarei-Hanzaki, H.R. Abedi, S. Mehtonen, D. Porter, Hot deformation characterization of duplex low-density steel through 3D processing map development, Mater. Charact. 107 (2015) 293-301.

[26] Y. Sun, L.X. Hu, J.S. Ren, Investigation on the hot deformation behavior of powder metallurgy TiAl-based alloy using 3D processing map, Mater. Charact. 100 (2015) 163-169.

[27] S. Zhang, F. Kong, Y. Chen, Z. Liu, J. Lin, Evolution of grain boundary and texture in as-forged Ti-45Al-9Nb-Y alloy during tensile test at different temperature, Intermetallics, 27 (2012) 31-37.

[28] H.Z. Niu, F.T. Kong, Y.Y. Chen, F. Yang, Microstructure characterization and tensile properties of $\beta$ phase containing TiAl pancake, J. Alloys Compd. 509 (2011) 10179-10184.

[29] H. Beladi, P. Cizek, P.D. Hodgson, Texture and substructure characteristics of dynamic recrystallization in a Ni$30 \% F e$ austenitic model alloy, Scr. Mater. 61 (2009) 528-531.

[30] Y. Zong, D. Wen, Z. Liu, D. Shan, Y-Phase transformation, dynamic recrystallization and texture of a forged TiAlbased alloy based on plane strain compression at elevated temperature, Mater. Des. 91 (2016) 321-330. 
[31] A. Momeni, G.R. Ebrahimi, M. Jahazi, P. Bocher, Microstructure evolution at the onset of discontinuous dynamic recrystallization: A physics-based model of subgrain critical size, J. Alloys Compd. 587 (2014) 199-210.

[32] F.J. Humphreys, A unified theory of recovery, recrystallization and grain growth, based on the stability and growth of cellular microstructures—I. The basic model, Acta Mater. 45 (1997) 4231-4240.

[33] Z. Jin, G.T.G. III, On deformation twins and twin-related lamellae in TiAl, J. Mater. Sci. 33 (1998) 77-83.

[34] Y. Cao, H. Di, J. Zhang, J. Zhang, T. Ma, R.D.K. Misra, An electron backscattered diffraction study on the dynamic recrystallization behavior of a nickel-chromium alloy $(800 \mathrm{H})$ during hot deformation, Mater. Sci. Eng., A, 585 (2013) $71-$ 85.

[35] D.P. Field, L.T. Bradford, M.M. Nowell, T.M. Lillo, The role of annealing twins during recrystallization of Cu, Acta Mater. 55 (2007) 4233-4241. 


\section{Highlights}

- The Ti-43Al-2Si alloy is prepared by cold crucible continuous casting, and the novel and effective method makes the alloy have excellent properties.

- The 3D processing map is developed for Ti-42Al-2Si alloy.

- The flow softening feature of this alloy is effect of dynamic recrystallization, globularization and reorientation of lamellae

- The discontinue dynamic recrystallization is deduced as the main dynamic recrystallization mechanism. 


\section{Table captions}

Table 1 The chemical compositions of different phases of Ti-43Al-2Si alloy ingot (at.\%). Table 2 Calculation results of deformation activation energy and material parameter. Table 3 The samples deformed under different processing condition.

Table 1 The chemical compositions of different phases of Ti-43Al-2Si alloy ingot (at.\%)

\begin{tabular}{cccc}
\hline Position & $\mathrm{Ti}$ & $\mathrm{Al}$ & $\mathrm{Si}$ \\
\hline $\mathrm{A}$ & 59.95 & 10.71 & 29.34 \\
$\mathrm{~B}$ & 51.75 & 47.55 & 0.71 \\
\hline
\end{tabular}

Table 2 Calculation results of deformation activation energy and material parameter

\begin{tabular}{cccc}
$\alpha$ & $\mathrm{n}$ & $\mathrm{Q}_{\mathrm{ave}} / \mathrm{kJ} / \mathrm{mol}$ & $\mathrm{A}$ \\
\hline 0.002861 & 4.293 & 451.1452 & $3.14 \times 10^{14}$ \\
\hline
\end{tabular}

Table 3 The samples deformed under different processing condition

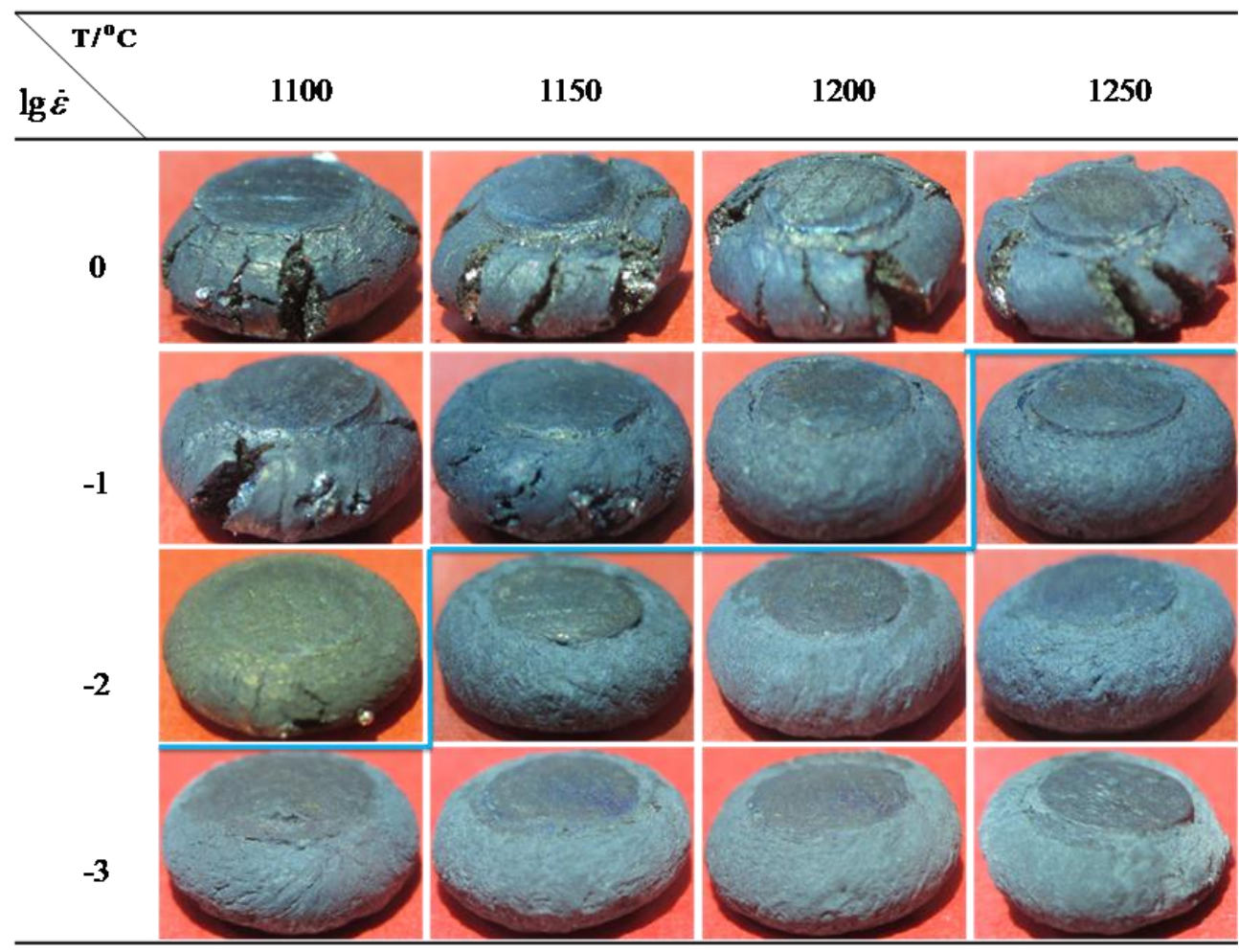




\section{Figure captions}

Fig. 1. Macrostructure and microstructure of the ingot: (a) low magnification, (b) high magnification by optical microscope, (c) microstructure by SEM-BSE, (d) unique grain color figure and pole figure by EBSD.

Fig. 2. Flow curves of the Ti-43Al-2Si alloy under different deformation conditions: (a) $1 \mathrm{~s}^{-1}$, (b) $0.1 \mathrm{~s}^{-1}$, (c) $0.01 \mathrm{~s}^{-1}$, (d) $0.001 \mathrm{~s}^{-1}$.

Fig. 3. The linear relationship: (a) $\ln \dot{\varepsilon}-\ln \sigma_{\mathrm{p}}$, (b) $\ln \dot{\varepsilon}-\sigma_{\mathrm{p}}$, (c) $\ln \left[\sinh \left(\alpha \sigma_{\mathrm{p}}\right)\right]-1000 / \mathrm{T}$, (d) $\ln \left[\sinh \left(\alpha \sigma_{\mathrm{p}}\right)\right]-\ln \dot{\varepsilon},(\mathrm{e}) \ln \mathrm{Z}-\ln \left[\sinh \left(\alpha \sigma_{\mathrm{p}}\right)\right]$.

Fig. 4. The 3D deformation activation energy map.

Fig. 5. The comparison of peak stress values from experiment and calculation.

Fig. 6. The $2 \mathrm{D}$ processing map of Ti-43Al-2Si alloy prepared by cold crucible continuous casting with at true strain of 0.8 (the shadow indicates the instability domain).

Fig. 7. Stress state of deformed sample.

Fig. 8. The 3D processing map: (a) power dissipation map of Ti-43Al-2Si alloy prepared by cold crucible continuous casting, (b) flow instability map of Ti-43Al-2Si alloy prepared by cold crucible continuous casting.

Fig. 9. Microstructure in the region III of Fig. 7 under different deformation conditions: (a) $1150^{\circ} \mathrm{C} / 1 \mathrm{~s}^{-1}$ (InZ=37.91), (b) $1200^{\circ} \mathrm{C} / 0.1 \mathrm{~s}^{-1}$ (InZ=34.32), (c) $1250^{\circ} \mathrm{C} / 0.01 \mathrm{~s}^{-1}$ (InZ=30.83).

Fig. 10. Microstructure in the region II of Fig. 7 under different deformation conditions: (a) $1150^{\circ} \mathrm{C} / 1 \mathrm{~s}^{-1}$ (InZ=37.91), (b) $1200^{\circ} \mathrm{C} / 0.1 \mathrm{~s}^{-1}$ (InZ=34.32), (c) $1250^{\circ} \mathrm{C} / 0.01 \mathrm{~s}^{-1}$ (InZ=30.83).

Fig. 11. UGCF and pole figures at strain rate of $0.01 \mathrm{~s}^{-1}$ and different temperature: (a) $1150^{\circ} \mathrm{C}(\ln Z=33.31),(b) 1250^{\circ} \mathrm{C}(\ln Z=30.83)$.

Fig. 12. Phase combined with rotation angle distribution map at strain rate of $0.01 \mathrm{~s}^{-1}$ and different temperature: (a) $1150^{\circ} \mathrm{C}(\ln Z=33.31)$, (b) $1250^{\circ} \mathrm{C}(\ln Z=30.83)$. 
Fig. 13. Misorientation angle distributions of Ti-43Al-2Si alloy at strain rate of $0.01 \mathrm{~s}^{-1}$ and different temperatures: (a) $1150^{\circ} \mathrm{C}(\ln Z=33.31)$, (b) $1250^{\circ} \mathrm{C}(\ln Z=30.83)$.

Fig. 14. GOS map of microstructural development at strain rate of $0.01 \mathrm{~s}^{-1}$ and different temperatures: (a) $1150^{\circ} \mathrm{C}(\ln Z=33.31),(b) 1250^{\circ} \mathrm{C}(\ln Z=30.83)$. 

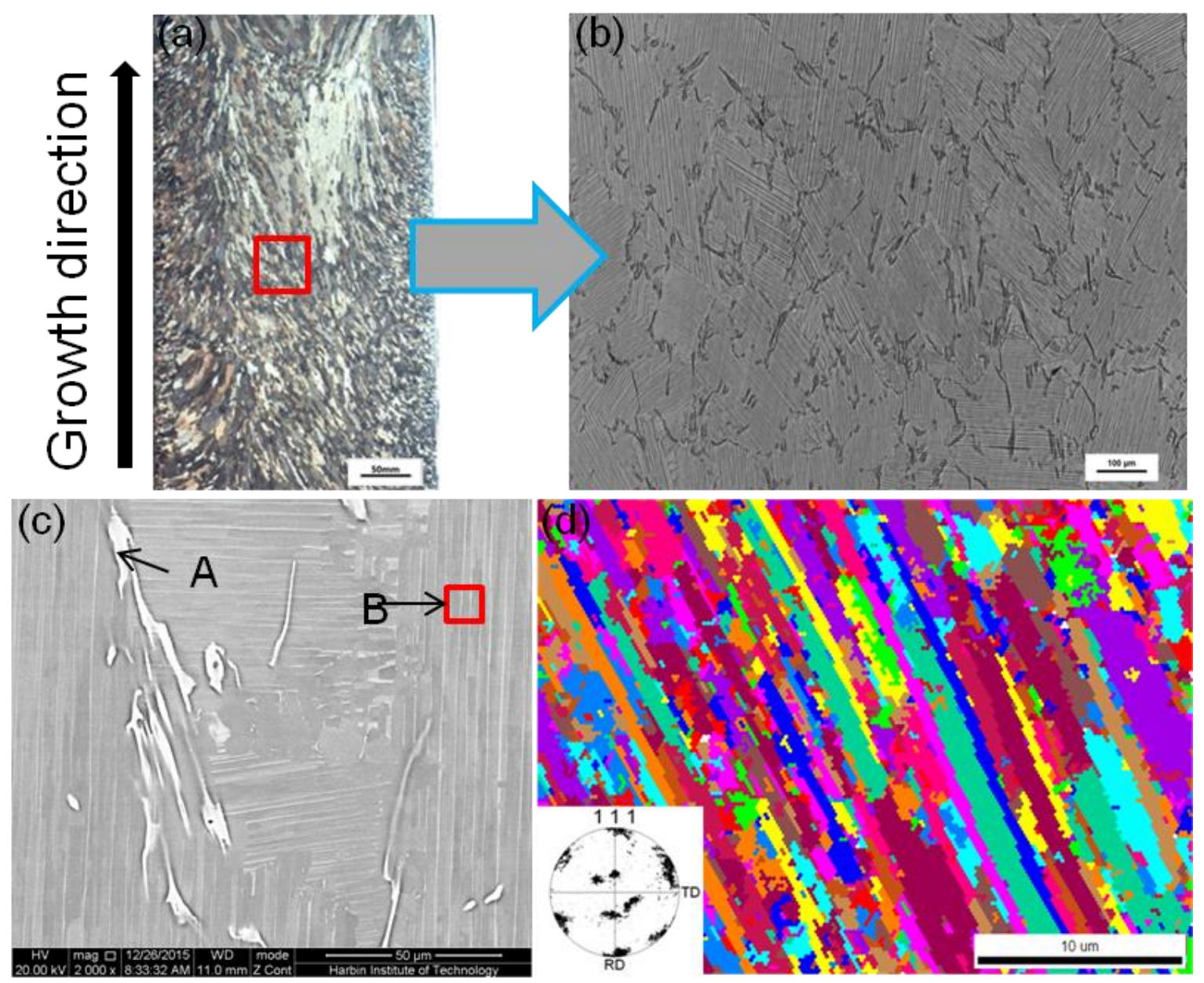

Fig.1. Macrostructure and microstructure of the ingot: (a) low magnification, (b) high magnification by optical microscope, (c) microstructure by SEM-BSE, (d) unique grain color figure and pole figure by EBSD. 

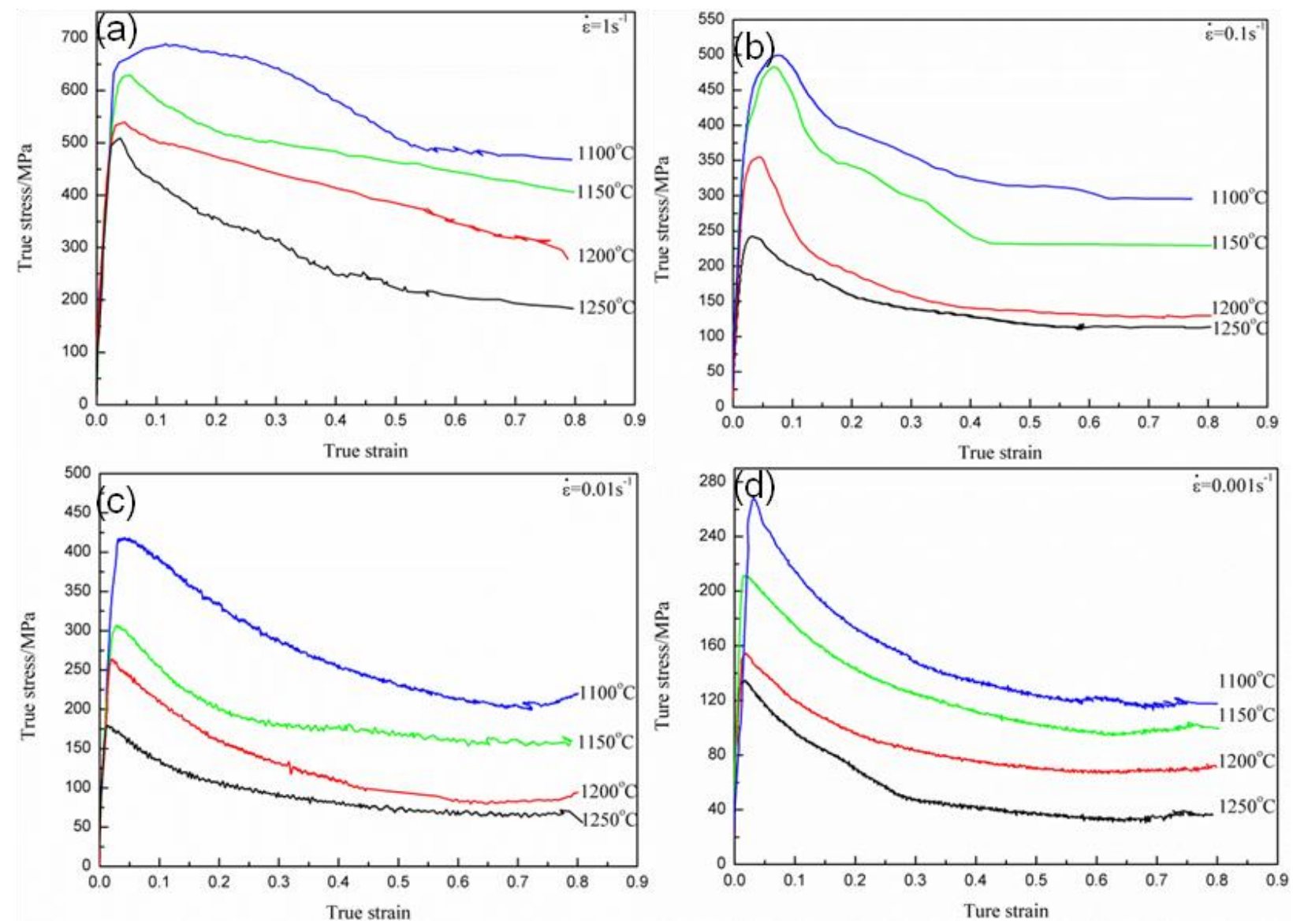

Fig.2. Flow curves of the Ti-43Al-2Si alloy under different deformation conditions: (a) $1 \mathrm{~s}^{-1}$, (b) $0.1 \mathrm{~s}^{-1}$, (c) $0.01 \mathrm{~s}^{-1}$, (d) $0.001 \mathrm{~s}^{-1}$. 

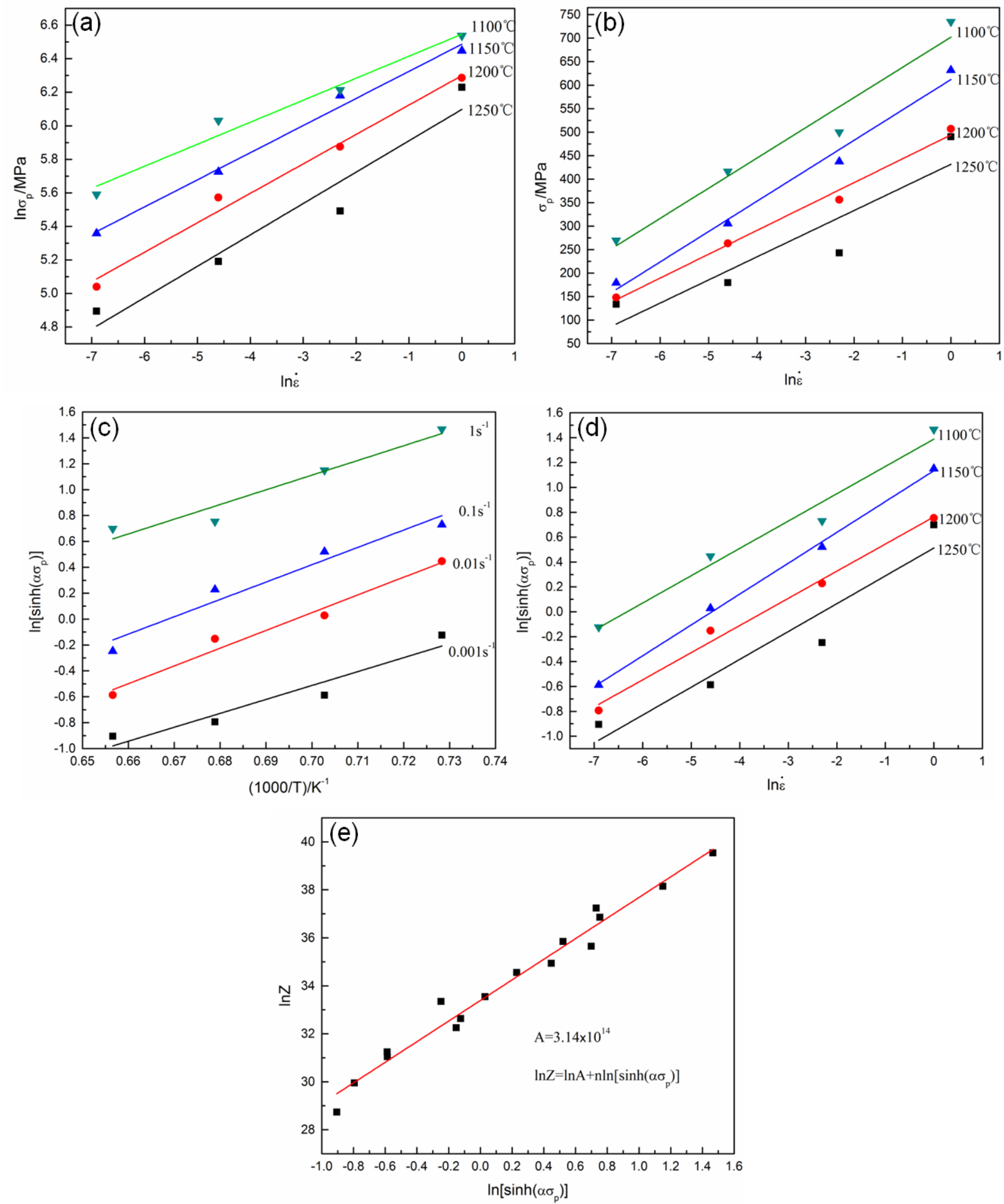

Fig. 3. The linear relationship: (a) $\ln \dot{\varepsilon}-\ln \sigma_{\mathrm{p}}$, (b) $\ln \dot{\varepsilon}-\sigma_{\mathrm{p}}$, (c) $\ln \left[\sinh \left(\alpha \sigma_{\mathrm{p}}\right)\right]-1000 / \mathrm{T}$, (d) $\ln \left[\sinh \left(\alpha \sigma_{\mathrm{p}}\right)\right]-\ln \dot{\varepsilon}$, (e) $\ln \mathrm{Z}-\ln \left[\sinh \left(\alpha \sigma_{\mathrm{p}}\right)\right]$. 


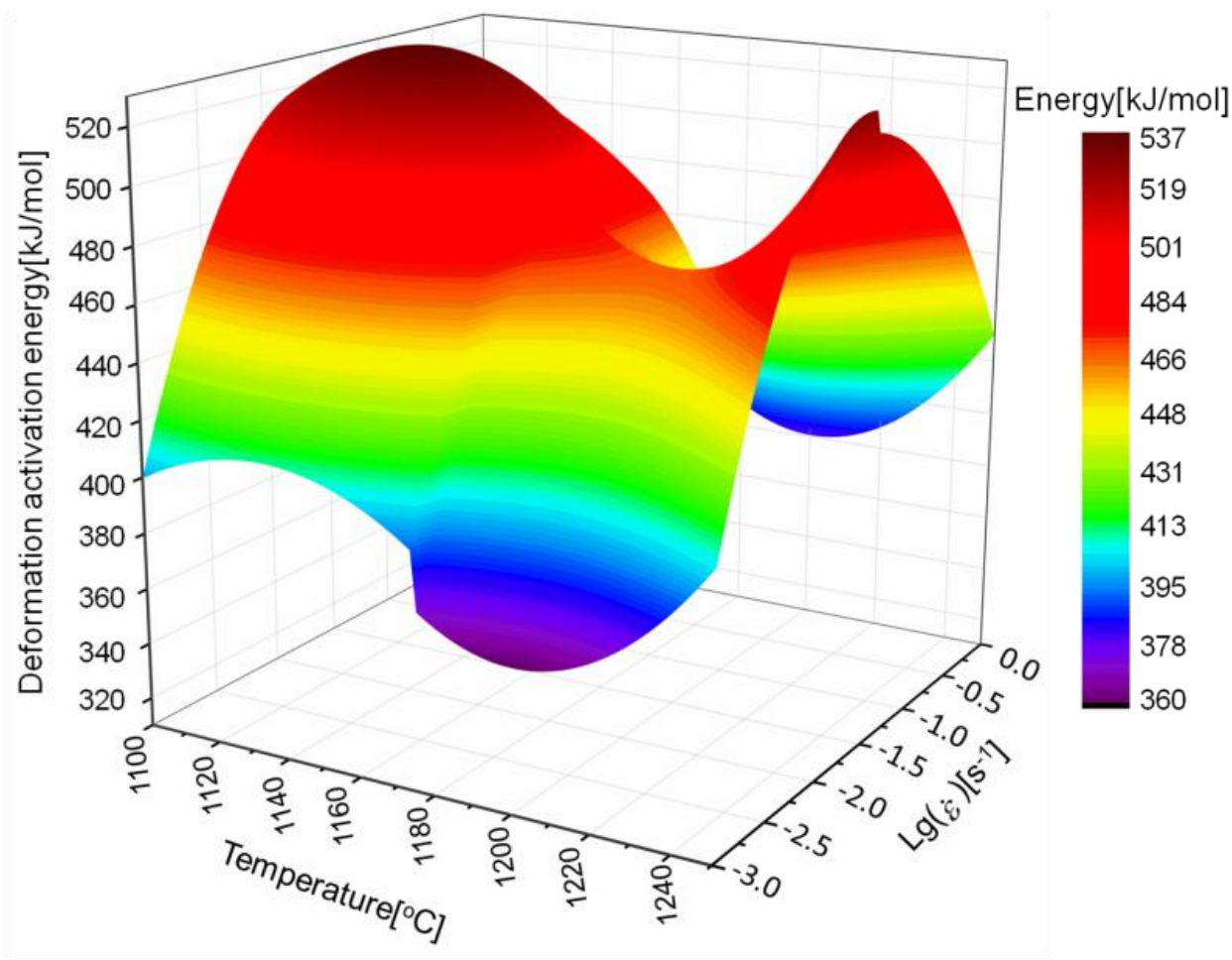

Fig. 4. The 3D deformation activation energy map.

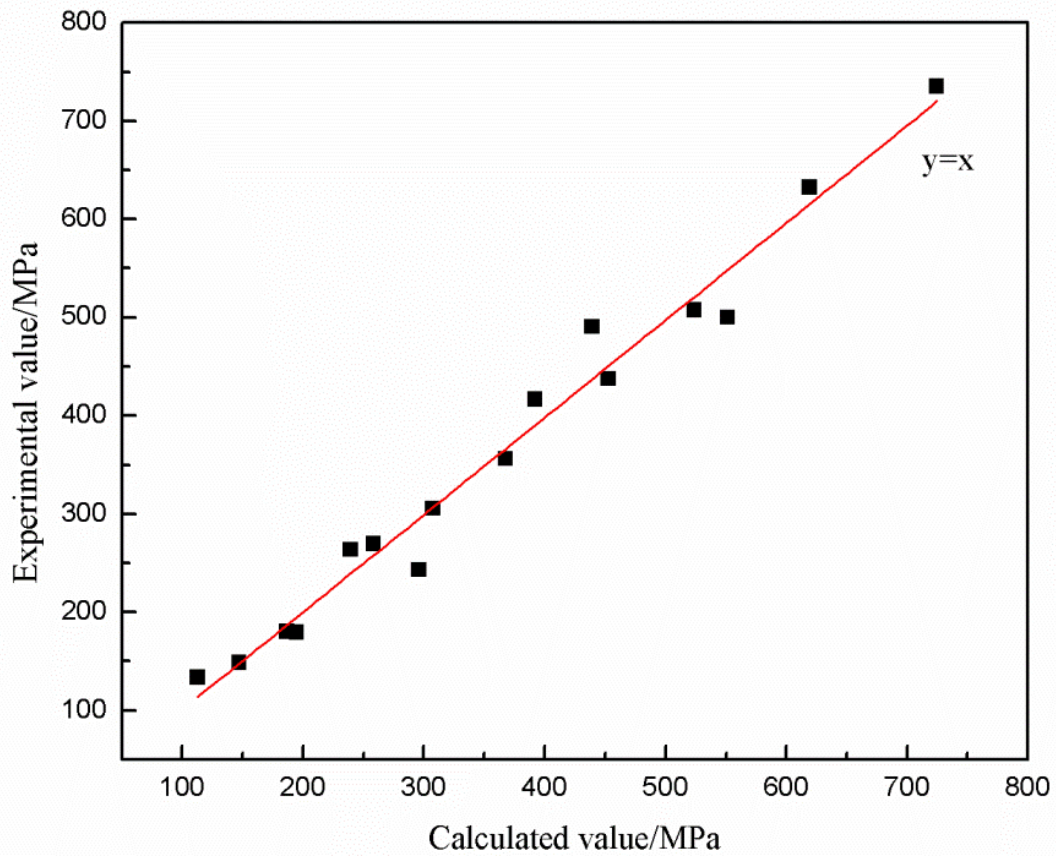

Fig. 5. The comparison of peak stress values from experiment and calculation. 


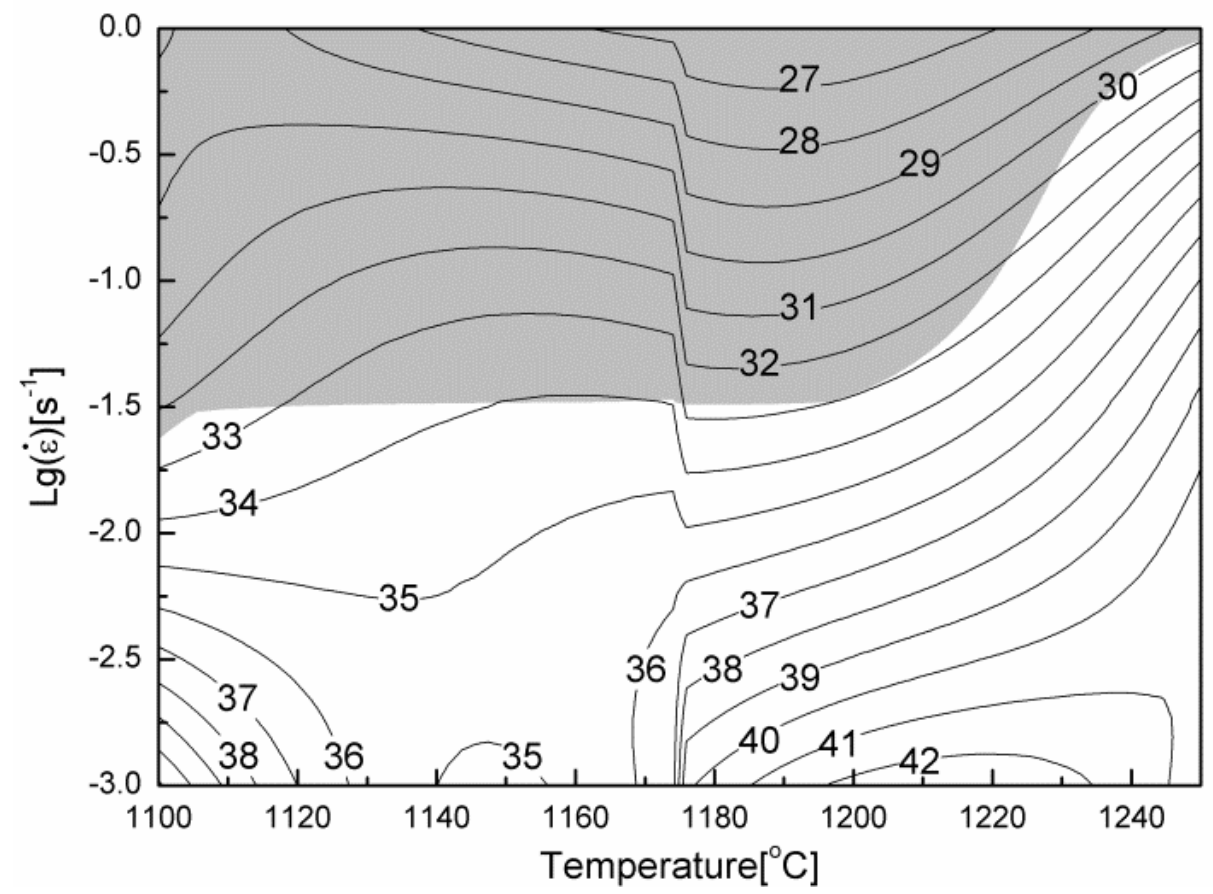

Fig. 6. The 2D processing map of Ti-43Al-2Si alloy prepared by cold crucible continuous castinG with at true strain of 0.8 (the shadow indicates the instability domain).

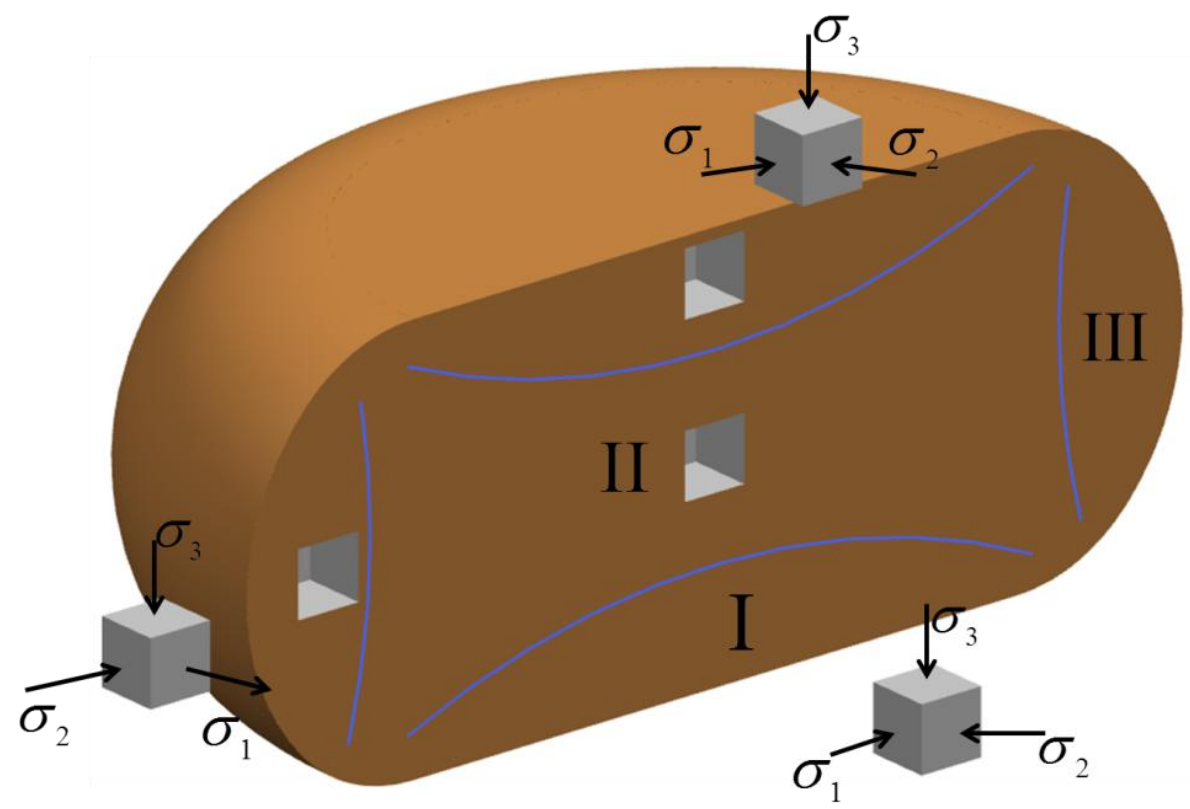

Fig. 7. Stress state of deformed sample. 

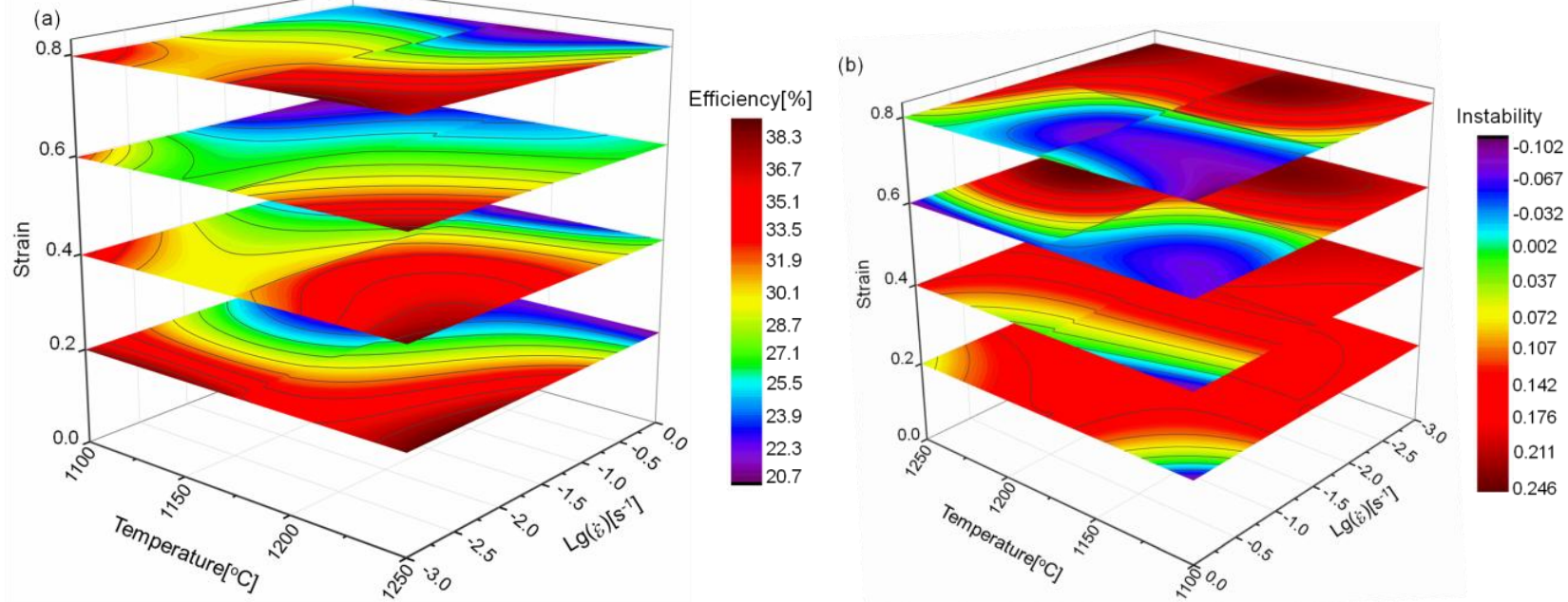

Fig. 8. The 3D processing map: (a) power dissipation map of Ti-43Al-2Si alloy prepared by cold crucible continuous casting, (b) flow instability map of Ti-43Al-2Si alloy prepared by cold crucible continuous casting.

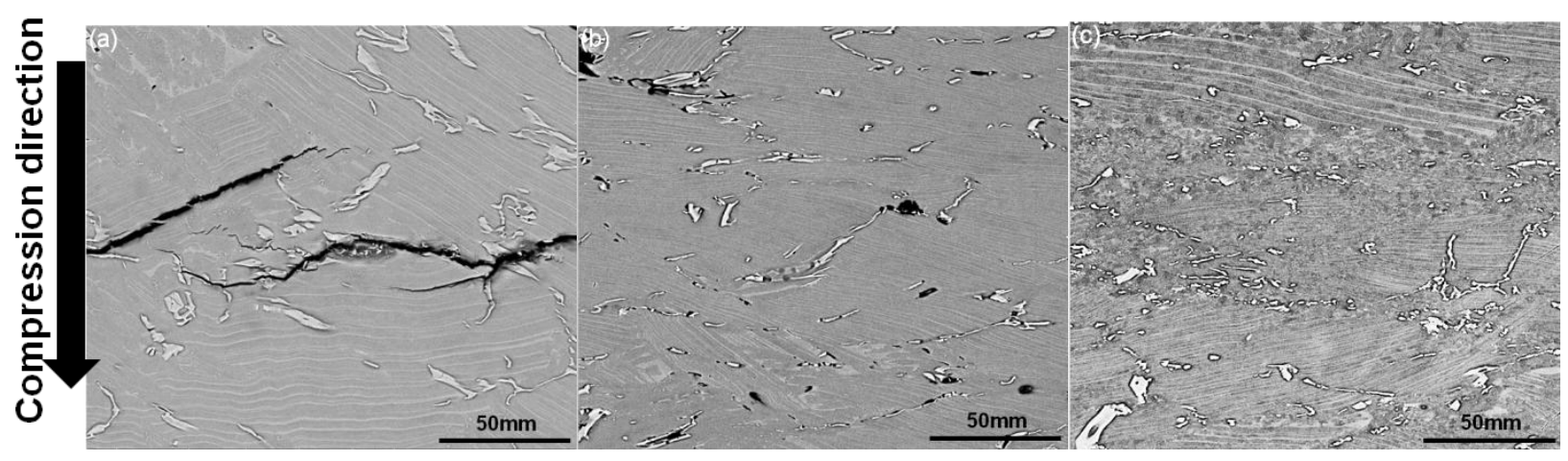

Fig. 9. Microstructure in the region III of Fig. 7 under different deformation conditions: (a) $1150^{\circ} \mathrm{C} / 1 \mathrm{~s}^{-1}$ (InZ=37.91), (b) $1200^{\circ} \mathrm{C} / 0.1 \mathrm{~s}^{-1}(\ln Z=34.32)$, (c) $1250^{\circ} \mathrm{C} / 0.01 \mathrm{~s}^{-1}(\ln Z=30.83)$.

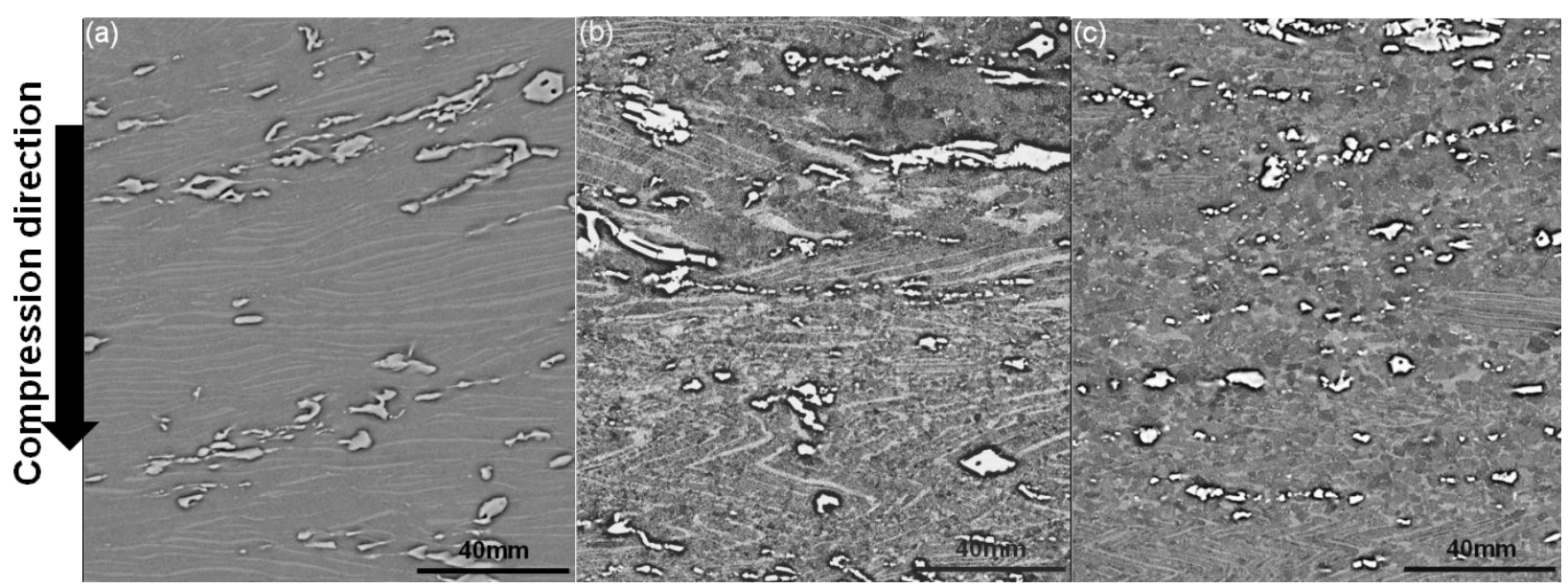

Fig. 10. Microstructure in the region II of Fig. 7 under different deformation conditions: (a) $1150^{\circ} \mathrm{C} / 1 \mathrm{~s}^{-1}$ (InZ=37.91), (b) $1200^{\circ} \mathrm{C} / 0.1 \mathrm{~s}^{-1}(\ln Z=34.32)$, (c) $1250^{\circ} \mathrm{C} / 0.01 \mathrm{~s}^{-1}(\ln Z=30.83)$. 


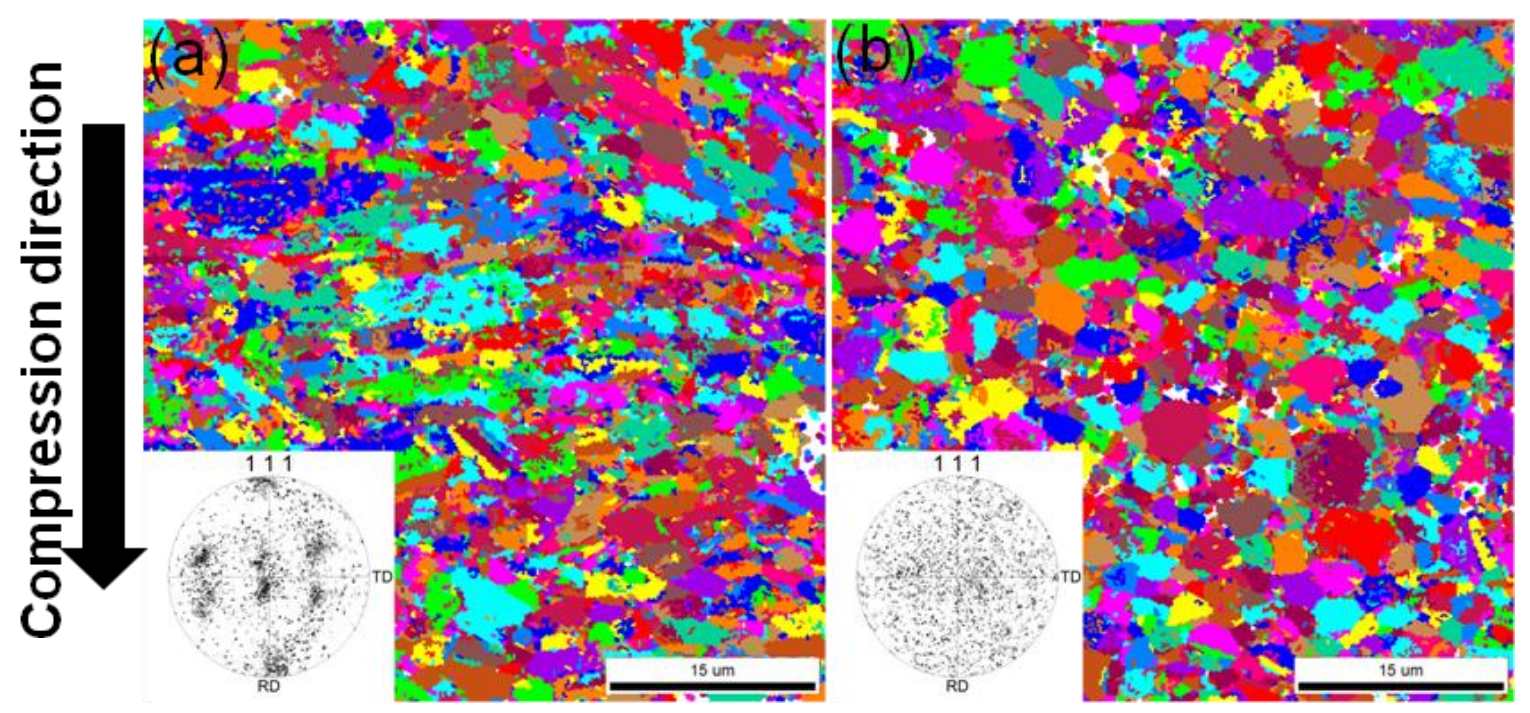

Fig. 11. UGCF and pole figures at strain rate of $0.01 \mathrm{~s}^{-1}$ and different temperature: (a) $1150^{\circ} \mathrm{C}$ (InZ=33.31), (b) $1250^{\circ} \mathrm{C}(\ln Z=30.83)$.

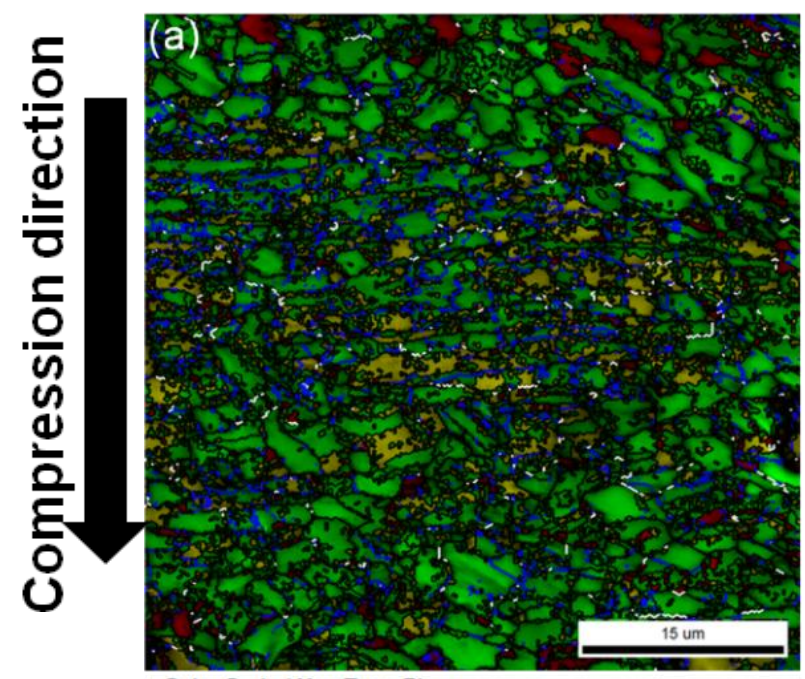

Color Coded Map Type: Phase

\begin{tabular}{|c|c|c|}
\hline & Total & Partition \\
\hline Phase & Fraction & Fraction \\
\hline$\overline{\text { Aluminum Titanium }}$ & 0.071 & 0.071 \\
\hline Titanium Aluminum & 0.726 & 0.726 \\
\hline Titanium Silicide & 0.203 & 0.203 \\
\hline
\end{tabular}

Boundaries: Rotation Angle

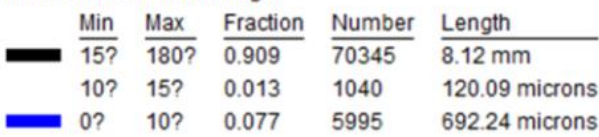

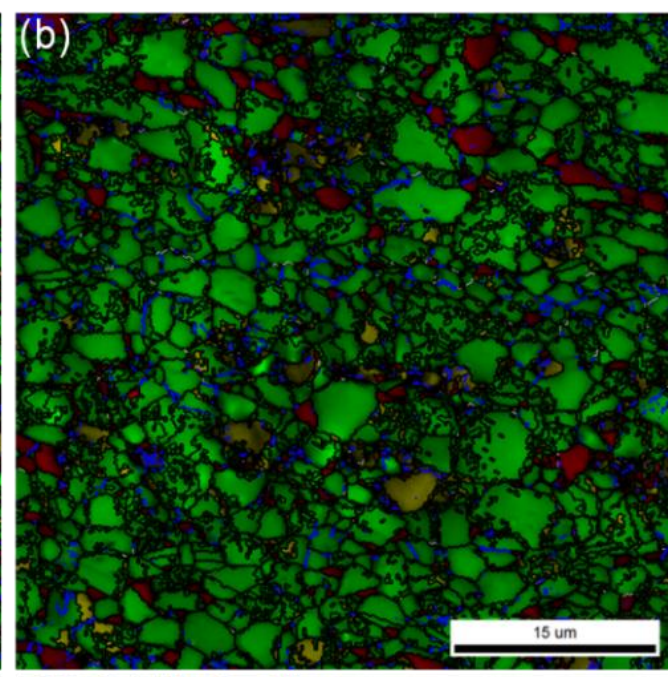

Color Coded Map Type: Phase

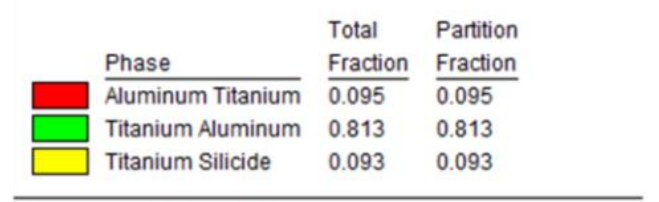

Boundaries: Rotation Angle

\begin{tabular}{|c|c|c|c|c|}
\hline Min & Max & Fraction & Number & Length \\
\hline$\overline{15 ?}$ & $\overline{180 ?}$ & 0.949 & 63219 & $7.30 \mathrm{~mm}$ \\
\hline $10 ?$ & $15 ?$ & 0.007 & 486 & 56.12 microns \\
\hline 0? & $10 ?$ & 0.043 & 2884 & 333.02 microns \\
\hline
\end{tabular}

Fig. 12. Phase combined with rotation angle distribution map at strain rate of $0.01 \mathrm{~s}^{-1}$ and different temperature: (a) $1150^{\circ} \mathrm{C}(\ln Z=33.31)$, (b) $1250^{\circ} \mathrm{C}(\ln Z=30.83)$. 

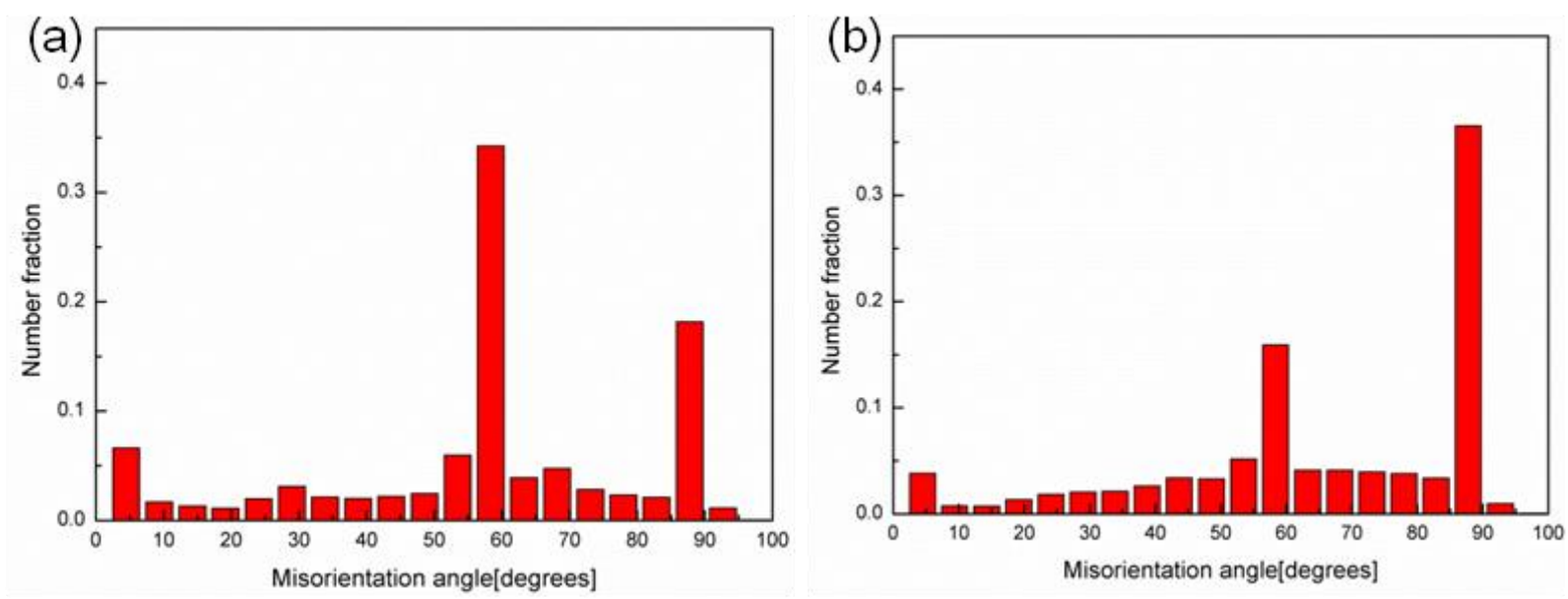

Fig. 13. Misorientation angle distributions of Ti-43Al-2Si alloy at strain rate of $0.01 \mathrm{~s}^{-1}$ and different temperatures: (a) $1150^{\circ} \mathrm{C}(\ln Z=33.31)$, (b) $1250^{\circ} \mathrm{C}(\ln Z=30.83)$.
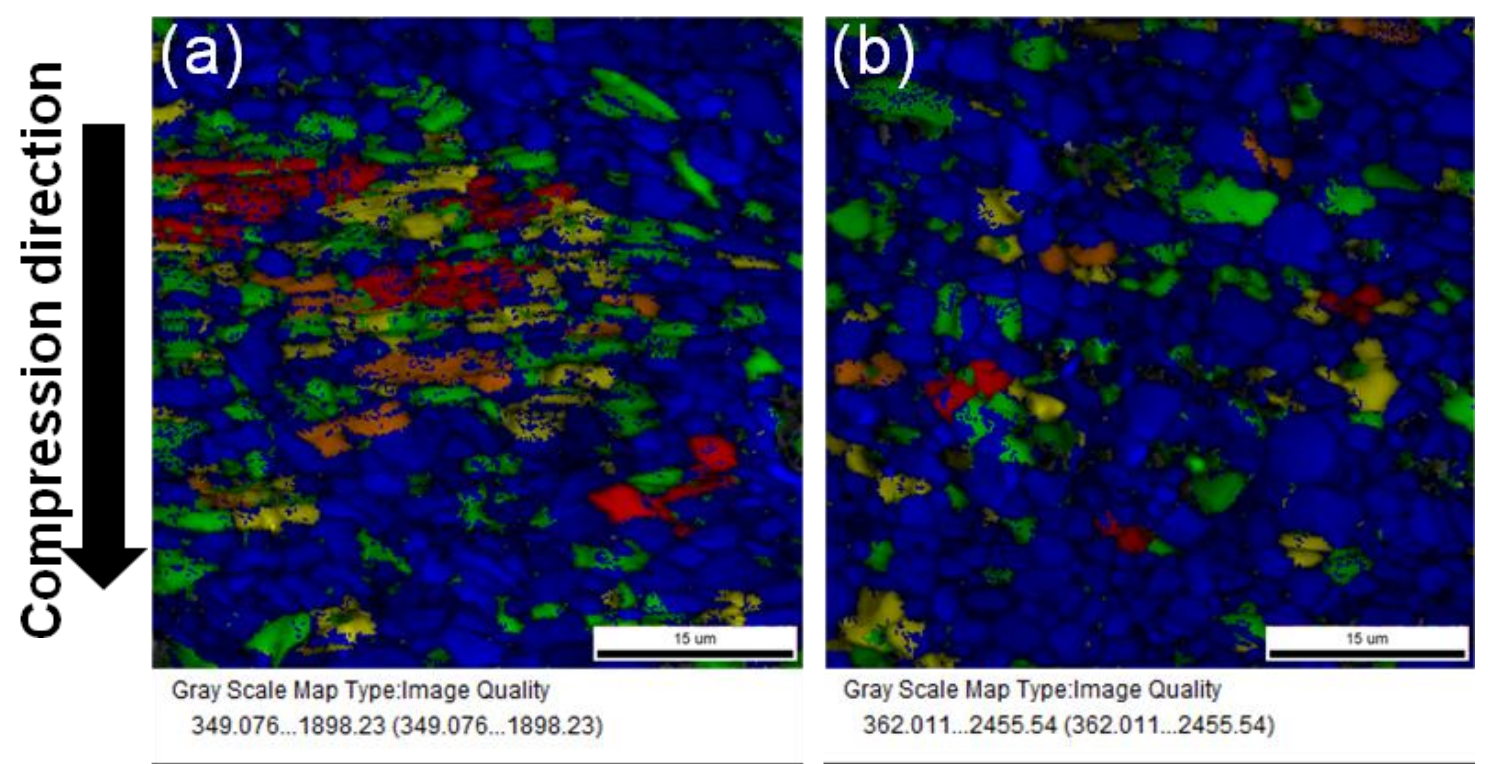

Color Coded Map Type: Grain Orientation Spread

Color Coded Map Type: Grain Orientation Spread

\begin{tabular}{|c|c|c|c|}
\hline Min & Max & $\begin{array}{l}\text { Total } \\
\text { Fraction }\end{array}$ & $\begin{array}{l}\text { Partition } \\
\text { Fraction }\end{array}$ \\
\hline$\overline{0}$ & $\overline{0.957124}$ & 0.691 & 0.691 \\
\hline 0.957124 & 1.91425 & 0.163 & 0.163 \\
\hline 1.91425 & 2.87137 & 0.062 & 0.062 \\
\hline 2.87137 & 3.8285 & 0.025 & 0.025 \\
\hline 3.8285 & 4.78562 & 0.051 & 0.051 \\
\hline
\end{tabular}

\begin{tabular}{|c|c|c|c|}
\hline Min & Max & $\begin{array}{l}\text { Total } \\
\text { Fraction }\end{array}$ & $\begin{array}{l}\text { Partition } \\
\text { Fraction }\end{array}$ \\
\hline 0 & 0.864792 & 0.796 & 0.796 \\
\hline 0.864792 & 1.72958 & 0.121 & 0.121 \\
\hline 1.72958 & 2.59437 & 0.047 & 0.047 \\
\hline 2.59437 & 3.45917 & 0.013 & 0.013 \\
\hline 3.45917 & 4.32396 & 0.011 & 0.011 \\
\hline
\end{tabular}

Fig. 14. GOS map of microstructural development at strain rate of $0.01 \mathrm{~s}^{-1}$ and different temperatures: (a) $1150^{\circ} \mathrm{C}(\ln Z=33.31),(b) 1250^{\circ} \mathrm{C}(\ln Z=30.83)$. 